\title{
Existence theorem for a unique solution to a coupled system of impulsive fractional differential equations in complex-valued fuzzy metric spaces
}

\author{
Humaira ${ }^{1}$, Hasanen A. Hammad ${ }^{2} \mathbb{D}$, Muhammad Sarwar $^{1 *}$ and Manuel De la Sen ${ }^{3}$
}

${ }^{*}$ Correspondence:

sarwarswati@gmail.com

1 Department of Mathematics,

University of Malakand, Chakdara Dir(L), Pakistan

Full list of author information is available at the end of the article

\section{Springer}

\begin{abstract}
In this manuscript, the existence theorem for a unique solution to a coupled system of impulsive fractional differential equations in complex-valued fuzzy metric spaces is studied and the fuzzy version of some fixed point results by using the definition and properties of a complex-valued fuzzy metric space is presented. Ultimately, some appropriate examples are constructed to illustrate our theoretical results.
\end{abstract}

Keywords: Complex-valued t-norm; Complex-valued fuzzy metric space; Common fixed point; Cauchy sequence; Coupled system of impulsive fractional differential equations

\section{Introduction}

Fuzzy sets theory plays an important role in our real life problems facing indeterminacy and vague situation. They allow us to model a very hard and uncertain situation in an easy way. This fact initiated an interesting research activity which led to the improvement of fuzzy set theory. The concept of fuzzy set was introduced by Zadeh where the association of an element to a set is expressed as a number from the interval [0,1] [1]. Afterward, Heilpern gave the concept of fuzzy mapping and obtained fixed point results in metric spaces, which signifies the fuzzy generalization of Banach contraction principle [2]. Several researchers studied fixed point theorems for fuzzy mappings via different types of contraction in several spaces, for example, see [3-6]. Furthermore, Kaleva and Seikkala realized that when the distance between the elements is not an exact number, then the imprecision is involved in the metric, and they introduced the concept of fuzzy metric spaces [7]. The concept of fuzzy metric was developed by Kramosil and Michalek [8] and further by George and Veeramani [9]. A variety of works have been done by many authors in regard of the existence results of fixed point (FP) in the fuzzy metric spaces (FMSs), for instance, we refer to [10-15].

In addition to the FMS, there are still several extensions of metric space terms in metric FP theory. Azam et al. [16] established a new approach by replacing the set of real numbers

(c) The Author(s) 2021. This article is licensed under a Creative Commons Attribution 4.0 International License, which permits use, sharing, adaptation, distribution and reproduction in any medium or format, as long as you give appropriate credit to the original author(s) and the source, provide a link to the Creative Commons licence, and indicate if changes were made. The images or other third party material in this article are included in the article's Creative Commons licence, unless indicated otherwise in a credit line to the material. If material is not included in the article's Creative Commons licence and your intended use is not permitted by statutory regulation or exceeds the permitted use, you will need to obtain permission directly from the copyright holder. To view a copy of this licence, visit http://creativecommons.org/licenses/by/4.0/. 
with the set of complex numbers endowed with an ordered structure. In this regard, they introduced the notion of complex-valued metric spaces (CVMSs). In recent years, Shukla et al. [17] extended the setting of FMSs to complex-valued fuzzy metric spaces (CVFMSs). They also obtained FP theorems of contractive mappings on CVFMSs.

FP theory has a wide range of applications in advanced mathematics. Banach contraction principle is a well-celebrated result in the analysis which is a widely used technique in mathematical analysis. Due to the simplicity of the Banach technique, it is the most valuable tool to solve several existing problems in mathematics. Several researchers generalized it in many directions satisfying different types of contractive conditions and discussed various applications in mathematical disciplines [18-25].

Fractional order derivative is an extension of integer order derivative that has been considered as a strong tool for modeling different mathematical problems. That is why fractional differential equations are considered as a significant and advanced area of applied mathematics, which has wide ranging application in different scientific disciplines like chemistry, physics, biology, and engineering. Thus, it has become an interesting and motivating area of research in the last few decades [26-32].

In the meanwhile, researchers claim that a complicated system and process cannot be described by a single differential equation. Therefore, the coupled systems containing fractional differential equations have also achieved farfetched attention, and thus many results are dedicated to them [33-39].

In the other direction impulsive fractional differential equations have had a rapid progress over the year. They perform a significant role to provide a natural framework for modeling of different real processes arising in phenomena discussed in various scientific fields, especially those problems which are subject to abrupt changes and discontinuous jumps and cannot be defined by the integer order differential equations. Thus, there is a variety of manuscripts available in the background literature in which several authors discussed impulsive fractional differential equations with different boundary conditions $(\mathrm{BC})$; for instance, we refer to [40-42] and the references cited therein.

Motivated by the above discussion, in this manuscript, some coupled fixed point consequences in the framework of complex-valued fuzzy metric spaces are proved. Thereafter, the theoretical results are involved to find the existence and uniqueness of a coupled system of nonlinear fractional differential equations with impulses in the form of

$$
\left\{\begin{array}{l}
{ }^{\mathrm{c}} \mathrm{D}^{\mu} \mathrm{x}(\mathrm{t})+\mathrm{h}\left(\mathrm{t},{ }^{\mathrm{c}} \mathrm{D}^{\varrho} \mathrm{x}(\mathrm{t}),{ }^{\mathrm{c}} \mathrm{D}^{\rho} \mathrm{y}(\mathrm{t})\right)=0 \quad \mathrm{t} \neq \mathrm{t}_{\mathrm{m}}, \mathrm{m}=1,2, \ldots, \mathrm{n}, \\
{ }^{\mathrm{c}} \mathrm{D}^{\gamma} \mathrm{y}(\mathrm{t})+\mathrm{g}\left(\mathrm{t},{ }^{\mathrm{c}} \mathrm{D}^{\varrho} \mathrm{y}(\mathrm{t}),{ }^{\mathrm{c}} \mathrm{D}^{\rho} \mathrm{x}(\mathrm{t})\right)=0 \quad \mathrm{t} \neq \mathrm{t}_{\mathrm{m}}, \mathrm{m}=1,2, \ldots, \mathrm{n}, \\
\left.\Delta \mathrm{x}\right|_{\mathrm{t}=\mathrm{t}_{\mathrm{m}}}=\mathcal{M}_{1 \mathrm{~m}}\left(\mathrm{x}\left(\mathrm{t}_{\mathrm{m}}\right)\right),\left.\Delta \mathrm{x}^{\prime}\right|_{\mathrm{t}=\mathrm{t}_{\mathrm{m}}}=\mathcal{N}_{1 \mathrm{~m}}\left(\mathrm{x}\left(\mathrm{t}_{\mathrm{m}}\right)\right),\left.\Delta \mathrm{x}^{\prime \prime}\right|_{\mathrm{t}=\mathrm{t}_{\mathrm{m}}}=\mathcal{O}_{1 \mathrm{~m}}\left(\mathrm{x}\left(\mathrm{t}_{\mathrm{m}}\right)\right) \\
\left.\Delta \mathrm{y}\right|_{\mathrm{t}=\mathrm{t}_{\mathrm{m}}}=\mathcal{M}_{2 \mathrm{~m}}\left(\mathrm{y}\left(\mathrm{t}_{\mathrm{m}}\right)\right),\left.\Delta \mathrm{y}^{\prime}\right|_{\mathrm{t}=\mathrm{t}_{\mathrm{m}}}=\mathcal{N}_{2 \mathrm{~m}}\left(\mathrm{y}\left(\mathrm{t}_{\mathrm{m}}\right)\right),\left.\Delta \mathrm{y}^{\prime \prime}\right|_{\mathrm{t}=\mathrm{t}_{\mathrm{m}}}=\mathcal{O}_{2 \mathrm{~m}}\left(\mathrm{y}\left(\mathrm{t}_{\mathrm{m}}\right)\right), \\
\mathrm{x}(0)=\mathrm{x}^{\prime}(0)=0,{ }^{c} \mathrm{D}^{5} \mathrm{x}(\tau)=\mathrm{x}^{\prime \prime}(1), \\
\mathrm{y}(0)=\mathrm{y}^{\prime}(0)=0,{ }^{\mathrm{c}} \mathrm{D}^{\kappa} \mathrm{y}(\epsilon)=\mathrm{y}^{\prime \prime}(1),
\end{array}\right.
$$

where $\mathrm{t} \in \mathrm{J}=[0,1], 2<\mu, \gamma \leq 3,0<\varrho, \rho, \varsigma, \tau, \kappa, \epsilon<1$. ${ }^{\mathrm{c}} \mathrm{D}$ stands for the Caputo fractional derivative and $\mathrm{h}, \mathrm{g}: \mathrm{J} \times \mathbb{R}^{3} \rightarrow \mathbb{R}$ are continuous functions. $\mathcal{M}_{1 \mathrm{~m}}, \mathcal{M}_{2 \mathrm{~m}}, \mathcal{N}_{1 \mathrm{~m}}, \mathcal{N}_{2 \mathrm{~m}}, \mathcal{O}_{1 \mathrm{~m}}$, $\mathcal{O}_{2 \mathrm{~m}} \in \mathrm{C}(\mathbb{R}, \mathbb{R})$ and $\mathrm{t}_{\mathrm{m}}$ satisfies $0=\mathrm{t}_{0}<\mathrm{t}<1<\cdots<\mathrm{t}_{\mathrm{n}}<\mathrm{t}_{\mathrm{n}+1}=1,\left.\Delta \mathrm{x}\right|_{\mathrm{t}=\mathrm{t}_{\mathrm{m}}}=\mathrm{x}\left(\mathrm{t}_{\mathrm{m}}^{+}\right)-\mathrm{x}\left(\mathrm{t}_{\mathrm{m}}^{-}\right)$, $\left.\Delta \mathrm{x}^{\prime}\right|_{\mathrm{t}=\mathrm{t}_{\mathrm{m}}}=\mathrm{x}^{\prime}\left(\mathrm{t}_{\mathrm{m}}^{+}\right)-\mathrm{x}^{\prime}\left(\mathrm{t}_{\mathrm{m}}^{-}\right),\left.\Delta \mathrm{x}^{\prime \prime}\right|_{\mathrm{t}=\mathrm{t}_{\mathrm{m}}}=\mathrm{x}^{\prime \prime}\left(\mathrm{t}_{\mathrm{m}}^{+}\right)-\mathrm{x}^{\prime \prime}\left(\mathrm{t}_{\mathrm{m}}^{-}\right),\left.\Delta \mathrm{y}\right|_{\mathrm{t}=\mathrm{t}_{\mathrm{m}}}=\mathrm{y}\left(\mathrm{t}_{\mathrm{m}}^{+}\right)-\mathrm{y}\left(\mathrm{t}_{\mathrm{m}}^{-}\right),\left.\Delta \mathrm{y}^{\prime}\right|_{\mathrm{t}=\mathrm{t}_{\mathrm{m}}}=$ 
$y^{\prime}\left(t_{m}^{+}\right)-y^{\prime}\left(t_{m}^{-}\right),\left.\Delta y^{\prime \prime}\right|_{t=t_{m}}=y^{\prime \prime}\left(t_{m}^{+}\right)-y^{\prime \prime}\left(t_{m}^{-}\right)$represent the right and left limits of $x(t), y(t)$, respectively, at $t=t_{m}$. Finally, nontrivial examples are presented to support our results.

\section{Preliminaries}

Throughout the paper, we denote the set of complex numbers by $\mathbb{C}$. Let $A=\{(x, y): 0 \leq x \leq$ $\infty, 0 \leq y \leq \infty\} \subset \mathbb{C}$. The elements $(0,0),(1,1) \in \mathbb{C}$ are denoted by $\vartheta$ and $v$, respectively. Let the unit closed complex interval be denoted by $\Xi=\{(z, y): 0 \leq z \leq 1,0 \leq y \leq 1\}$, the open unit complex interval be denoted by $\Xi_{0}=\{(z, y): 0 \leq z<1,0 \leq y<1\}$, and the set $\{(z, y): 0<z<\infty, 0<y<\infty\}$ be denoted by $A_{\vartheta}$.

Let $\Omega \subset \mathbb{C}$. If there exists inf $\Omega$ so that the lower bound of $\Omega$ is inf $\Omega \preceq c, \forall c \in \Omega$ and $\mathbf{v} \preceq \inf \Omega$ for every lower bound $\mathbf{v}$ of $\Omega$, then $\inf \Omega$ is called the greatest lower bound of $\Omega$. In the same way we define sup $\Omega$, (lub) the least upper bound of $\Omega$.

Definition 2.1 ([17]) Let $\partial$ be a nonempty set. A complex fuzzy set $\nabla$ is characterized by a mapping with domain $\partial$ and values in the closed complex unit interval $\Xi$.

Definition 2.2 ([17]) A binary relation $\star: \Re \times \Re \rightarrow \Re$ is called a complex-valued $t$-norm if, for all $\xi, \xi^{1}, \xi^{2}, \xi^{3}, \xi^{3} \in \Re$, the stipulations below hold.

$\left(\mathrm{t}_{1}\right) \xi^{1} \star \xi^{2}=\xi^{2} \star \xi^{1}$;

(t $\left.\mathrm{t}_{2}\right)$ if $\xi^{1} \prec \xi^{3}$ and $\xi^{2} \prec \xi^{4}$, then $\xi^{1} \star \xi^{2} \prec \xi^{3} \star \xi^{4}$;

$\left(\mathrm{t}_{3}\right) \xi^{1} \star\left(\xi^{2} \star \xi^{3}\right)=\left(\xi^{1} \star \xi^{2}\right) \star \xi^{3}$;

$\left(\mathrm{t}_{3}\right) \xi \star \vartheta=\vartheta$ and $\xi \star v=v$.

Definition 2.3 ([17]) Assume that $\partial$ is a nonempty set, $\star$ is a continuous complex-valued $\omega$-norm, $\nabla$ is a complex fuzzy set on $\partial \times \partial \times A_{\vartheta} \rightarrow O$ verifying the stipulations below: For each $\xi^{1}, \xi^{2}, \xi^{3} \in \partial, \omega, \varpi>A_{\vartheta}$,

$\left(C b M F_{1}\right) \nabla\left(\xi^{1}, \xi^{2}, \omega\right) \succ 0$

$\left(C b M F_{2}\right) \nabla\left(\xi^{1}, \xi^{2}, \omega\right)=v$ iff $\xi^{1}=\xi^{2}$

$\left(C b M F_{3}\right) \nabla\left(\xi^{1}, \xi^{2}, \omega\right)=\nabla\left(\xi^{2}, \xi^{1}, \omega\right)$;

$\left(C b M F_{4}\right) \nabla\left(\xi^{1}, \xi^{2}, \omega\right) \star \nabla\left(\xi^{2}, \xi^{3}, \varpi\right) \preceq \nabla\left(\xi^{1}, \xi^{3}, \omega+\varpi\right) ;$

$\left(\mathrm{CbMF}_{5}\right) \nabla\left(\xi^{1}, \xi^{2}, \star\right): A_{\vartheta} \rightarrow O$ is continuous.

We say that a trio $(\partial, \nabla, \star)$ is a CVFMS and $\nabla$ is called a CVFM on $\partial$.

The function $\nabla\left(\xi^{1}, \xi^{2}, \omega\right)$ stands for the degree of nearness and the degree of nonnearness between $\xi^{1}$ and $\xi^{2}$ with respect to (w.r.t.) the complex parameter $\omega$, respectively.

Example 2.1 Consider an arbitrary metric space $(\circlearrowright, \sigma)$. Define $\star$ by $\xi^{1} \star \xi^{2}=\left(e_{1} e_{2}, c_{1}, c_{2}\right)$ for all $\xi^{1}=\left(e_{1}, c_{1}\right), \xi^{2}=\left(e_{2}, c_{2}\right) \in \Xi$. Let the complex fuzzy set $\nabla$ be defined by

$$
\nabla(\mathrm{z}, \mathrm{y}, \omega)=\frac{e_{1}+c_{1}}{e_{1}+c_{1}+\sigma(\mathrm{z}, \mathrm{y})} v
$$

for all $\mathrm{z}, \mathrm{y} \in A_{\vartheta}, \omega=(e, c)$. Then $(\partial, \nabla, \star)$ is a CVFMS.

Indeed, in the above example, if $g: A_{\vartheta} \rightarrow(0, \infty)$ is a continuous and nondecreasing function, that is, $\xi^{1} \preceq \xi^{2}$ implies that $g\left(\xi^{1}\right) \leq g\left(\xi^{2}\right)$, then $(\partial, \nabla, \star)$ is a CVFMS, where

$$
\nabla(\mathrm{z}, \mathrm{y}, \omega)=\frac{g(\omega)}{g(\omega)+\sigma(\mathrm{z}, \mathrm{y})} v .
$$


Similarly it is obvious that, for the fuzzy set defined by

$$
\nabla(\mathrm{z}, \mathrm{y}, \omega)=\left[\exp \left(\frac{\sigma(\mathrm{z}, \mathrm{y})}{g(\omega)}\right)\right]^{-1} v
$$

$(\supset, \nabla, \star)$ is a CVFMS.

Definition 2.4 ([17]) Let $(\partial, \nabla, \star)$ be a CVFMS. A sequence $\left\{\theta_{m}\right\} \in \partial$ converges to $\theta \in \partial$ if for each $r \in \Xi_{0}$ and $\omega \in A_{\vartheta}$ there exists $k_{0} \in \mathbb{N}$ with

$$
v-\mathrm{r} \prec \nabla\left(\theta_{m}, \theta, \omega\right) \quad \forall m>k_{0} .
$$

Definition 2.5 ([17]) Let $(\partial, \nabla, \star)$ be a CVFMS. A sequence $\left\{\theta_{m}\right\}$ in $\circlearrowright$ is known as a Cauchy sequence if

$$
\lim _{m \rightarrow \infty} \inf _{n>m} \nabla\left(\theta_{m}, \theta_{n}, \omega\right)=v \quad \forall \omega \in A_{\vartheta} .
$$

The CVFMS $(\partial, \nabla, \star)$ is called complete if every Cauchy sequence is convergent in $\partial$.

Lemma 2.6 ([17]) Let $(\partial, \nabla, \star)$ be a CVFMS. If $\omega, \omega^{\prime} \in A_{\vartheta}$ and $\omega \preceq \omega^{\prime}$, then $\nabla(\mathrm{z}, \mathrm{y}, \omega) \preceq$ $\nabla\left(\mathrm{z}, \mathrm{y}, \omega^{\prime}\right) \forall \mathrm{z}, \mathrm{y} \in \partial$.

Remark 2.7 ([17]) Assume that $\theta_{m} \in A$ for each $m \in \mathbb{N}$.

(1) If there exist $\alpha, \beta \in A$ with $\alpha \prec \theta_{m} \prec \beta$ and the sequence $\left\{\theta_{m}\right\}$ is monotonic with respect to $\prec$ for each $m \in \mathbb{N}$, then there is $\theta \in A$ so that $\lim _{m \rightarrow \infty} \theta_{m}=\theta$

(2) The pair $(\mathbb{C}, \prec$ ) is a lattice in spite of $\prec$ being not a linear order on $\mathbb{C}$.

Remark 2.8 ([17]) Assume that $\theta_{m}, \theta_{m}^{*}, u \in A, \forall m \in \mathbb{N}$.

- If $\theta_{m} \prec \theta_{m}^{*} \prec v, \forall m \in \mathbb{N}$ and $\lim _{m \rightarrow \infty} \theta_{m}=v$, then $\lim _{m \rightarrow \infty} \theta_{m}^{*}=v$.

- If $\theta_{m} \prec u, \forall m \in \mathbb{N}$ and $\lim _{m \rightarrow \infty} \theta_{m}=\theta$, then $\theta \prec u$.

- If $u \prec \theta_{m}, \forall m \in \mathbb{N}$ and $\lim _{m \rightarrow \infty} \theta_{m}=\theta$, then $u \prec \theta$.

Lemma $2.9([17])$ Assume that $(\partial, \nabla, \star)$ is a CVFMS. A sequence $\left\{\theta_{m}\right\} \in \partial$ converges to $j \in \supset$ if $\lim _{m \rightarrow \infty} \nabla\left(\theta_{m}, j, \omega\right)=v$ holds for all $\omega \in A_{\vartheta}$.

Definition $2.10([43])$ Assume that $\partial \neq \emptyset$. We say the two mappings $\Lambda: \partial \times \partial \rightarrow \partial$ and $\eta: \partial \rightarrow \partial$ are commutative if $\eta\left(\Lambda\left(c^{1}, c^{2}\right)\right)=\Lambda\left(\eta c^{1}, \eta c^{2}\right)$.

\section{Coupled fixed point results}

Let $\Phi$ denote the class of all functions $\phi:[0,1] \rightarrow[0,1]$ such that $\phi$ is increasing, continuous, and let $\phi(t)>t$ for all $t \in(0,1)$. Note that if $\phi(0)=0$ and $\phi(1)=1$ additionally hold, then $\phi(t) \geq t, t \in[0,1]$ for all functions in $\Phi$.

Now we shall discuss the existence and uniqueness of a coupled common fixed point (CCFP) for two-self mappings in the setting of CVFMSs by introducing the two theorems below. 
Theorem 3.1 Let $(\partial, \nabla, \star)$ be a complete CVFMS, $\Lambda: \partial \times \partial \rightarrow \partial$ and $\eta: \partial \rightarrow \partial$ be selfmappings so that

$$
\left(v-\nabla\left(\Lambda\left(c^{1}, c^{2}\right), \Lambda\left(b^{1}, b^{2}\right), \omega\right)\right) \preceq \mu\left(v-\max \left\{\nabla\left(\eta c^{1}, \eta b^{1}, \omega\right), \nabla\left(\eta c^{2}, \eta b^{2}, \omega\right)\right\}\right)
$$

for each $c^{1}, c^{2}, b^{1}, b^{2} \in \nabla, \omega \in A_{\vartheta}$, where $\mu \in(0,1]$. If the pair mapping $(\Lambda, \eta)$ satisfies the stipulations below:

(i) $\Lambda(\partial \times \partial) \subseteq \eta(\partial)$;

(ii) $\eta(\partial)$ is complete;

(iii) $\eta$ is continuous and commutes with $\Lambda$.

Then $\Lambda$ and $\eta$ have a unique CCFP.

Proof Let $c_{\circ}^{1}, c_{\circ}^{2} \in \partial$. Because $\Lambda(\partial \times \partial) \subseteq \eta(\partial)$, one can select $c_{1}^{1}, c_{1}^{2} \in \partial$ such that $\eta\left(c_{\circ}^{1}\right)=$ $\Lambda\left(c_{\circ}^{1}, c_{\circ}^{2}\right)$ and $\eta\left(c_{\circ}^{2}\right)=\Lambda\left(c_{\circ}^{2}, c_{\circ}^{1}\right)$. Again by stipulation (i), we can choose $c_{2}^{1}, c_{2}^{2} \in \partial$ such that $\eta\left(c_{2}^{1}\right)=\Lambda\left(c_{1}^{1}, c_{1}^{2}\right)$ and $\eta\left(c_{2}^{2}\right)=\Lambda\left(c_{1}^{2}, c_{1}^{1}\right)$. By continuing the same logic reasoning, we can build two sequences $\left\{c_{m}^{1}\right\},\left\{c_{m}^{2}\right\} \in \partial$ so that

$$
\eta\left(c_{m+1}^{1}\right)=\Lambda\left(c_{m}^{1}, c_{m}^{2}\right) \quad \text { and } \quad \eta\left(c_{m+1}^{2}\right)=\Lambda\left(c_{m}^{2}, c_{m}^{1}\right) \quad \text { for } m \in \mathbb{N}_{\circ} .
$$

According to (3.1), we have

$$
\begin{aligned}
(v & \left.-\nabla\left(\eta\left(c_{m-1}^{1}\right), \eta\left(c_{m}^{1}\right), \omega\right)\right) \\
& =\left(v-\nabla\left(\Lambda\left(c_{m-2}^{1}, c_{m-2}^{2}\right), \Lambda\left(c_{m-1}^{1}, c_{m-1}^{2}\right), \omega\right)\right) \\
& \leq \mu\left(v-\max \left\{\nabla\left(\eta c_{m-2}^{1}, \eta c_{m-1}^{1}, \omega\right), \nabla\left(\eta c_{m-2}^{2}, \eta c_{m-1}^{2}, \omega\right)\right\}\right) \\
& \preceq v-\max \left\{\nabla\left(\eta c_{m-2}^{1}, \eta c_{m-1}^{1}, \omega\right), \nabla\left(\eta c_{m-2}^{2}, \eta c_{m-1}^{2}, \omega\right)\right\},
\end{aligned}
$$

this leads to

$$
\nabla\left(\eta\left(c_{m-1}^{1}\right), \eta\left(c_{m}^{1}\right), \omega\right) \succeq \max \left\{\nabla\left(\eta c_{m-2}^{1}, \eta c_{m-1}^{1}, \omega\right), \nabla\left(\eta c_{m-2}^{2}, \eta c_{m-1}^{2}, \omega\right)\right\}
$$

similarly,

$$
\begin{aligned}
(v & \left.-\nabla\left(\eta\left(c_{m-1}^{2}\right), \eta\left(c_{m}^{2}\right), \omega\right)\right) \\
& =\left(v-\nabla\left(\Lambda\left(c_{m-2}^{2}, c_{m-2}^{1}\right), \Lambda\left(c_{m-1}^{2}, c_{m-1}^{1}\right), \omega\right)\right) \\
& \preceq \mu\left(v-\max \left\{\nabla\left(\eta c_{m-2}^{2}, \eta c_{m-1}^{2}, \omega\right), \nabla\left(\eta c_{m-2}^{1}, \eta c_{m-1}^{1}, \omega\right)\right\}\right) \\
& \preceq v-\max \left\{\nabla\left(\eta c_{m-2}^{2}, \eta c_{m-1}^{2}, \omega\right), \nabla\left(\eta c_{m-2}^{1}, \eta c_{m-1}^{1}, \omega\right)\right\},
\end{aligned}
$$

which leads to

$$
\nabla\left(\eta\left(c_{m-1}^{2}\right), \eta\left(c_{m}^{2}\right), \omega\right) \succeq \max \left\{\nabla\left(\eta c_{m-2}^{2}, \eta c_{m-1}^{2}, \omega\right), \nabla\left(\eta c_{m-2}^{1}, \eta c_{m-1}^{1}, \omega\right)\right\}
$$

Using (3.3) and (3.5), we get

$$
\begin{aligned}
\theta_{m} & =\max \left\{\nabla\left(\eta\left(c_{m-1}^{1}\right), \eta\left(c_{m}^{1}\right), \omega\right), \nabla\left(\eta\left(c_{m-1}^{2}\right), \eta\left(c_{m}^{2}\right), \omega\right)\right\} \\
& \geq \max \left\{\nabla\left(\eta c_{m-2}^{1}, \eta c_{m-1}^{1}, \omega\right), \nabla\left(\eta c_{m-2}^{2}, \eta c_{m-1}^{2}, \omega\right)\right\}=\theta_{m-1}
\end{aligned}
$$


for each $m \in \mathbb{N}$. Now, we want to show that $\theta_{m}=v$. Hence, by the definition, we have

$$
v \succeq\left\{\theta_{m}\right\} \succeq\left\{\theta_{m-1}\right\} \succeq \vartheta
$$

Thus, $\left\{\theta_{m}(\omega)\right\}$ is a monotonic sequence in $A$. By (3.5) and Remark 2.7, there is $v^{*} \in A$ so that

$$
\lim _{m \rightarrow \infty}\left\{\theta_{m}\right\}=v^{*}
$$

Inequality (3.2) suggests that

$$
(1-\mu) v+\mu \max \left\{\nabla\left(\eta c_{m-2}^{1}, \eta c_{m-1}^{1}, \omega\right), \nabla\left(\eta c_{m-2}^{2}, \eta c_{m-1}^{2}, \omega\right)\right\} \preceq \nabla\left(\eta\left(c_{m-1}^{1}\right), \eta\left(c_{m}^{1}\right), \omega\right),
$$

or

$$
(1-\mu) v+\mu \theta_{m-1} \preceq \nabla\left(\eta\left(c_{m-1}^{1}\right), \eta\left(c_{m}^{1}\right), \omega\right),
$$

similarly, inequality (3.4) leads to

$$
(1-\mu) v+\mu \theta_{m-1} \preceq \nabla\left(\eta\left(c_{m-1}^{2}\right), \eta\left(c_{m}^{2}\right), \omega\right) .
$$

It follows from (3.8) and (3.9) that

$$
(1-\mu) v+\mu \theta_{m-1} \preceq \max \left\{\nabla\left(\eta\left(c_{m-1}^{1}\right), \eta\left(c_{m}^{1}\right), \omega\right), \nabla\left(\eta\left(c_{m-1}^{2}\right), \eta\left(c_{m}^{2}\right), \omega\right)\right\}=\theta_{m},
$$

this yields $(1-\mu) v \preceq \theta_{m}-\mu \theta_{m-1}$. By (3.7), we can write

$$
(1-\mu) v \preceq(1-\mu) \nu^{*}
$$

Because $\mu \in(0,1]$ and utilizing Remark 2.8, we obtain that $v=v^{*}$. Thus

$$
\lim _{m \rightarrow \infty}\left\{\theta_{m}\right\}=v .
$$

Next, we show that $\left\{\eta c_{m}^{1}\right\}$ and $\left\{\eta c_{m}^{2}\right\}$ are Cauchy sequences in $\eta(\circlearrowright)$ for $m \in \mathbb{N}$. First we prove that, for each $v$, there are two numbers $n, m \in \mathbb{N}$ so that

$$
\max \left\{\nabla\left(\eta c_{m}^{1}, \eta c_{n}^{1}, \omega\right), \nabla\left(\eta c_{m}^{2}, \eta c_{n}^{2}, \omega\right)\right\} \succ v
$$

Assume the contrary. Thus, for each $v$ and integer $\sigma$, there exist integers $n(\sigma)$ and $m(\sigma)$ with $m(\sigma)>n(\sigma) \geq \sigma$ so that

$$
\max \left\{\nabla\left(\eta c_{m(\sigma)}^{1}, \eta c_{n(\sigma)}^{1}, \omega\right), \nabla\left(\eta c_{m(\sigma)}^{2}, \eta c_{n(\sigma)}^{2}, \omega\right)\right\} \preceq \nu .
$$

\section{Consider}

$$
\max \left\{\nabla\left(\eta c_{m(\sigma)}^{1}, \eta c_{n(\sigma)-1}^{1}, \omega\right), \nabla\left(\eta c_{m(\sigma)}^{2}, \eta c_{n(\sigma)-1}^{2}, \omega\right)\right\} \succ v
$$


Assume that $n(\sigma)$ is the smallest number exceeding $m(\sigma)$ so that (3.10) holds. Let

$$
\varkappa_{\sigma}(\omega)=\max \left\{\nabla\left(\eta c_{m(\sigma)}^{1}, \eta c_{n(\sigma)}^{1}, \omega\right), \nabla\left(\eta c_{m(\sigma)}^{2}, \eta c_{n(\sigma)}^{2}, \omega\right)\right\}
$$

By (3.10), and using the fact for each $z_{1}, z_{2}, z_{3}, z_{4} \in \Xi, z_{1} \star z_{2} \preceq \max \left\{z_{1}, z_{3}\right\} \star \max \left\{z_{2}, z_{4}\right\}$, we can write

$$
\begin{aligned}
v \succeq & \varkappa_{\sigma}(\omega) \succeq \max \left\{\begin{array}{c}
\nabla\left(\eta c_{m(\sigma)}^{1}, \eta c_{n(\sigma)-1}^{1}, \frac{\omega}{2}\right) \star \nabla\left(\eta c_{n(\sigma)-1}^{1}, \eta c_{n(\sigma)}^{1} \frac{\omega}{2}\right), \\
\nabla\left(\eta c_{m(\sigma)}^{2}, \eta c_{n(\sigma)-1}^{2}, \frac{\omega}{2}\right) \star \nabla\left(\eta c_{n(\sigma)-1}^{2}, \eta c_{n(\sigma)}^{2}, \frac{\omega}{2}\right)
\end{array}\right\} \\
\succeq & \max \left\{\max \left\{\nabla\left(\eta c_{m(\sigma)}^{1}, \eta c_{n(\sigma)-1}^{1}, \omega\right), \nabla\left(\eta c_{m(\sigma)}^{2}, \eta c_{n(\sigma)-1}^{2}, \omega\right)\right\}\right. \\
& \star \max \left\{\nabla\left(\eta c_{n(\sigma)-1}^{1}, \eta c_{n(\sigma)}^{1} \frac{\omega}{2}\right), \nabla\left(\eta c_{n(\sigma)-1}^{2}, \eta c_{n(\sigma)}^{2}, \frac{\omega}{2}\right)\right\}, \\
& \max \left\{\nabla\left(\eta c_{m(\sigma)}^{1}, \eta c_{n(\sigma)-1}^{1}, \omega\right), \nabla\left(\eta c_{m(\sigma)}^{2}, \eta c_{n(\sigma)-1}^{2}, \omega\right)\right\} \\
& \left.\star \max \left\{\nabla\left(\eta c_{n(\sigma)-1}^{1}, \eta c_{n(\sigma)}^{1} \frac{\omega}{2}\right), \nabla\left(\eta c_{n(\sigma)-1}^{2}, \eta c_{n(\sigma)}^{2}, \frac{\omega}{2}\right)\right\}\right\} . \\
\succeq & \max \left\{\nabla\left(\eta c_{m(\sigma)}^{1}, \eta c_{n(\sigma)-1}^{1}, \omega\right), \nabla\left(\eta c_{m(\sigma)}^{2}, \eta c_{n(\sigma)-1}^{2}, \omega\right)\right\} \star \theta_{n(\sigma)}\left(\frac{\omega}{2}\right) .
\end{aligned}
$$

Taking $\sigma \rightarrow \infty$ in the above inequality, we get

$$
v \succeq \lim _{\sigma \rightarrow \infty} \varkappa_{\sigma}(\omega) \succeq v \star \theta_{n(\sigma)}\left(\frac{\omega}{2}\right)=v \star v=v .
$$

Thus,

$$
\lim _{\sigma \rightarrow \infty} \varkappa_{\sigma}(\omega)=v, \forall \omega \in A_{\vartheta}
$$

On the other hand, we get

$$
\begin{aligned}
& \varkappa_{\sigma}(\omega) \\
& \succeq \max \left\{\begin{array}{l}
\nabla\left(\eta c_{m(\sigma)}^{1}, \eta c_{m(\sigma)+1}^{1}, \frac{\omega}{3}\right) \star \nabla\left(\eta c_{m(\sigma)+1}^{1}, \eta c_{n(\sigma)+1}^{1}, \frac{\omega}{3}\right) \star \nabla\left(\eta c_{n(\sigma)+1}^{1}, \eta c_{n(\sigma)}^{1}, \frac{\omega}{3}\right), \\
\nabla\left(\eta c_{m(\sigma)}^{2}, \eta c_{m(\sigma)+1}^{2}, \frac{\omega}{3}\right) \star \nabla\left(\eta c_{m(\sigma)+1}^{2}, \eta c_{n(\sigma)+1}^{2}, \frac{\omega}{3}\right) \star \nabla\left(\eta c_{n(\sigma)+1}^{2}, \eta c_{n(\sigma)}^{2}, \frac{\omega}{3}\right)
\end{array}\right\}
\end{aligned}
$$

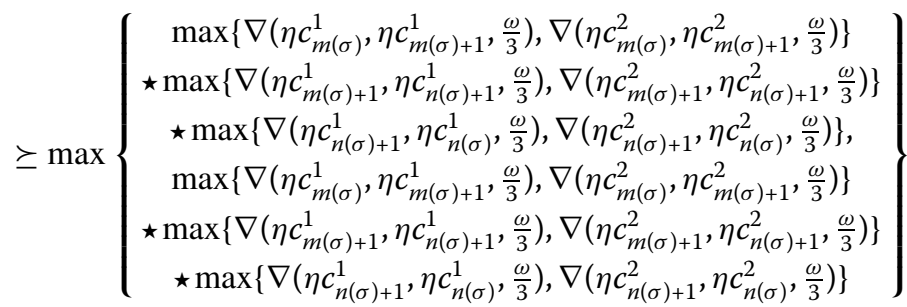

$$
\begin{aligned}
& =\max \left\{\nabla\left(\eta c_{m(\sigma)}^{1}, \eta c_{m(\sigma)+1}^{1}, \frac{\omega}{3}\right), \nabla\left(\eta c_{m(\sigma)}^{2}, \eta c_{m(\sigma)+1}^{2}, \frac{\omega}{3}\right)\right\} \\
& \star \max \left\{\nabla\left(\eta c_{m(\sigma)+1}^{1}, \eta c_{n(\sigma)+1}^{1}, \frac{\omega}{3}\right), \nabla\left(\eta c_{m(\sigma)+1}^{2}, \eta c_{n(\sigma)+1}^{2}, \frac{\omega}{3}\right)\right\} \\
& \star \max \left\{\nabla\left(\eta c_{n(\sigma)+1}^{1}, \eta c_{n(\sigma)}^{1}, \frac{\omega}{3}\right), \nabla\left(\eta c_{n(\sigma)+1}^{2}, \eta c_{n(\sigma)}^{2}, \frac{\omega}{3}\right)\right\}
\end{aligned}
$$




$$
\begin{aligned}
& =\theta_{m(\sigma)}\left(\frac{\omega}{3}\right) \star \max \left\{\nabla\left(\eta c_{m(\sigma)+1}^{1}, \eta c_{n(\sigma)+1}^{1}, \frac{\omega}{3}\right), \nabla\left(\eta c_{m(\sigma)+1}^{2}, \eta c_{n(\sigma)+1}^{2}, \frac{\omega}{3}\right)\right\} \\
& \star \theta_{n(\sigma)}\left(\frac{\omega}{3}\right) .
\end{aligned}
$$

Applying stipulation (3.1), we have

$$
\begin{aligned}
\varkappa_{\sigma}(\omega) & \geq \theta_{m(\sigma)}\left(\frac{\omega}{3}\right) \star \max \left\{\begin{array}{l}
\nabla\left(\Lambda\left(c_{m(\sigma)}^{1}, c_{m(\sigma)}^{2}\right), \Lambda\left(c_{n(\sigma)}^{1}, c_{n(\sigma)}^{2}\right), \frac{\omega}{3}\right), \\
\nabla\left(\Lambda\left(c_{m(\sigma)}^{2}, c_{m(\sigma)}^{1}\right), \Lambda\left(c_{n(\sigma)}^{2}, c_{n(\sigma)}^{1}\right), \frac{\omega}{3}\right)
\end{array}\right\} \star \theta_{n(\sigma)}\left(\frac{\omega}{3}\right) \\
& \succeq \theta_{m(\sigma)}\left(\frac{\omega}{3}\right) \star \max \left\{\nabla\left(\eta c_{m(\sigma)}^{1}, \eta c_{n(\sigma)}^{1}, \frac{\omega}{3}\right), \nabla\left(\eta c_{m(\sigma)}^{2}, \eta c_{n(\sigma)}^{2}, \frac{\omega}{3}\right)\right\} \star \theta_{n(\sigma)}\left(\frac{\omega}{3}\right) \\
& =\theta_{m(\sigma)}\left(\frac{\omega}{3}\right) \star \varkappa_{\sigma}(\omega) \star \theta_{n(\sigma)}\left(\frac{\omega}{3}\right) .
\end{aligned}
$$

Applying the limit as $\sigma \rightarrow \infty$ in (3.10), and by Definition 2.2, we can write

$$
v \succeq v \star v \star v=v,
$$

this is a contradiction. Thus $\left\{\eta c_{m}^{1}\right\}$ and $\left\{\eta c_{m}^{2}\right\}$ are Cauchy sequences in $\eta(\partial)$. The completeness of $\eta(\partial)$ and Lemma 2.9 leads to there are two elements $c^{1}, c^{2} \in \partial$ such that

$$
\lim _{a \rightarrow \infty} \nabla\left(\eta c_{m}^{1}, c^{1}, \omega\right)=v \quad \text { and } \quad \lim _{a \rightarrow \infty} \nabla\left(\eta c_{m}^{2}, c^{2}, \omega\right)=v \quad \text { for each } \omega \in A_{0}
$$

Also, the continuity of $\eta$ implies that $\left\{\eta \eta c_{m}^{1}\right\}$ and $\left\{\eta \eta c_{m}^{2}\right\}$ are convergent to $\eta c^{1}$ and $\eta c^{2}$ respectively. Moreover, since $\Lambda$ and $\eta$ commute, then one can write

$$
\eta \eta c_{m+1}^{1}=\eta\left(\Lambda\left(c_{m}^{1}, c_{m}^{2}\right)\right)=\Lambda\left(\eta c_{m}^{1}, \eta c_{m}^{2}\right)
$$

and

$$
\eta \eta c_{m+1}^{2}=\eta\left(\Lambda\left(c_{m}^{2}, c_{m}^{1}\right)\right)=\Lambda\left(\eta c_{m}^{2}, \eta c_{m}^{1}\right)
$$

Thus, by (3.1), we get

$$
\begin{aligned}
\nu-\nabla\left(\eta \eta c_{m+1}^{1}, \Lambda\left(c^{1}, c^{2}\right), \omega\right) & =v-\nabla\left(\Lambda\left(\eta c_{m}^{1}, \eta c_{m}^{2}\right), \Lambda\left(c^{1}, c^{2}\right), \omega\right) \\
& \preceq \mu\left(\nu-\max \left\{\nabla\left(\eta \eta c_{m}^{1}, \eta c^{1}, \omega\right), \nabla\left(\eta \eta c_{m}^{2}, \eta c^{2}, \omega\right)\right\}\right) \\
& \preceq v-\max \left\{\nabla\left(\eta \eta c_{m}^{1}, \eta c^{1}, \omega\right), \nabla\left(\eta \eta c_{m}^{2}, \eta c^{2}, \omega\right)\right\} .
\end{aligned}
$$

This leads to

$$
\nabla\left(\eta \eta c_{m+1}^{1}, \Lambda\left(c^{1}, c^{2}\right), \omega\right) \succeq \max \left\{\nabla\left(\eta \eta c_{m}^{1}, \eta c^{1}, \omega\right), \nabla\left(\eta \eta c_{m}^{2}, \eta c^{2}, \omega\right)\right\}
$$

Applying the limit as $m \rightarrow \infty$, we have

$$
\nabla\left(\eta c^{1}, \Lambda\left(c^{1}, c^{2}\right), \omega\right)=v
$$


Hence $\eta c^{1}=\Lambda\left(c^{1}, c^{2}\right)$, similarly, one can show that $\eta c^{2}=\Lambda\left(c^{2}, c^{1}\right)$. Thus the pair $\left(c^{1}, c^{2}\right)$ is a coupled FP of the mappings $\eta$ and $\Lambda$. Again, by (3.1), we get

$$
\begin{aligned}
v-\nabla\left(c^{1}, \eta c^{1}, \omega\right) & =\lim _{m \rightarrow \infty}\left(v-\nabla\left(\eta c_{m+1}^{1}, \eta c^{1}, \omega\right)\right) \\
& =\lim _{m \rightarrow \infty}\left(v-\nabla\left(\Lambda\left(c_{m}^{1}, c_{m}^{2}\right), \Lambda\left(c^{1}, c^{2}\right), \omega\right)\right) \\
& \preceq \lim _{m \rightarrow \infty}\left(\mu\left[v-\max \left\{\nabla\left(\eta c_{m}^{1}, \eta c^{1}, \omega\right), \nabla\left(\eta c_{m}^{2}, \eta c^{2}, \omega\right)\right\}\right]\right) \\
& =\mu\left[v-\max \left\{\nabla\left(c^{1}, \eta c^{1}, \omega\right), \nabla\left(c^{2}, \eta c^{2}, \omega\right)\right\}\right] \\
& \preceq v-\max \left\{\nabla\left(c^{1}, \eta c^{1}, \omega\right), \nabla\left(c^{2}, \eta c^{2}, \omega\right)\right\},
\end{aligned}
$$

this implies that

$$
\nabla\left(c^{1}, \eta c^{1}, \omega\right) \succeq \max \left\{\nabla\left(c^{1}, \eta c^{1}, \omega\right), \nabla\left(c^{2}, \eta c^{2}, \omega\right)\right\},
$$

similarly, one can write

$$
\nabla\left(c^{2}, \eta c^{2}, \omega\right) \succeq \max \left\{\nabla\left(c^{2}, \eta c^{2}, \omega\right), \nabla\left(c^{1}, \eta c^{1}, \omega\right)\right\} .
$$

Thus

$$
\max \left\{\nabla\left(c^{1}, \eta c^{1}, \omega\right), \nabla\left(c^{2}, \eta c^{2}, \omega\right)\right\} \succeq \max \left\{\nabla\left(c^{1}, \eta c^{1}, \omega\right), \nabla\left(c^{2}, \eta c^{2}, \omega\right)\right\}
$$

Hence $\nabla\left(c^{1}, \eta c^{1}, \omega\right)=v$ and $\nabla\left(c^{2}, \eta c^{2}, \omega\right)=v$. Thus $c^{1}=\eta c^{1}$ and $c^{2}=\eta c^{2}$ or, equivalently, $c^{1}=\eta c^{1}=\Lambda\left(c^{1}, c^{2}\right)$ and $c^{2}=\eta c^{2}=\Lambda\left(c^{2}, c^{1}\right)$.

For the uniqueness, assume that $c^{* 1} \in \partial$ with $c^{* 1} \neq c^{1}$ so that

$$
c^{* 1}=\eta c^{* 1}=\Lambda\left(c^{* 1}, c^{* 2}\right) .
$$

Then, by (3.1), we have

$$
\begin{aligned}
\nu-\nabla\left(c^{1}, c^{* 1}, \omega\right) & =v-\nabla\left(\Lambda\left(c^{1}, c^{2}\right), \Lambda\left(c^{* 1}, c^{* 2}\right), \omega\right) \\
& \preceq \mu\left(v-\max \left\{\nabla\left(\eta c^{1}, \eta c^{* 1}, \omega\right), \nabla\left(\eta c^{2}, \eta c^{* 2}, \omega\right)\right\}\right) \\
& \preceq v-\max \left\{\nabla\left(c^{1}, c^{* 1}, \omega\right), \nabla\left(c^{1}, c^{* 1}, \omega\right)\right\},
\end{aligned}
$$

which leads to $c^{1}=c^{* 1}$. So $\Lambda$ and $\eta$ have a unique CCFP.

Theorem 3.2 Assume that $(\partial, \nabla, \star)$ is a complete CVFMS, and let $\Lambda: \partial \times \partial \rightarrow \partial$ and $\eta: \partial \rightarrow \partial$ be self-mappings so that

$$
\nabla\left(\Lambda\left(c^{1}, c^{2}\right), \Lambda\left(b^{1}, b^{2}\right), \omega\right) \succeq \varphi\left(\min \left\{\nabla\left(\eta c^{1}, \eta b^{1}, \omega\right), \nabla\left(\eta c^{2}, \eta b^{2}, \omega\right)\right\}\right)
$$

for all $c^{1}, c^{2}, b^{1}, b^{2} \in \partial, \omega \in A_{\vartheta}$. Assume that $\Lambda$ and $\eta$ satisfy the same stipulations of Theorem 3.1 with $\varphi \in \Phi$. Then $\Lambda$ and $\eta$ have a unique CCFP provided that $\lim _{m \rightarrow \infty} \nabla\left(c_{m}^{1}, c_{m-1}^{1}\right.$, $\omega)=v$. 
Proof Assume that $\left\{c_{m}^{1}\right\}$ and $\left\{c_{m}^{2}\right\}$ are two sequences built in the proof of Theorem 3.1 so that

$$
\eta\left(c_{m+1}^{1}\right)=\Lambda\left(c_{m}^{1}, c_{m}^{2}\right) \text { and } \eta\left(c_{m+1}^{2}\right)=\Lambda\left(c_{m}^{2}, c_{m}^{1}\right) \quad \text { for } m \in \mathbb{N}_{\circ}
$$

It follows from (3.1) that

$$
\begin{aligned}
\nabla\left(\eta\left(c_{m-1}^{1}\right), \eta\left(c_{m}^{1}\right), \omega\right) & =\nabla\left(\Lambda\left(c_{m-2}^{1}, c_{m-2}^{2}\right), \Lambda\left(c_{m-1}^{1}, c_{m-1}^{2}\right), \omega\right) \\
& \geq \varphi\left(\min \left\{\nabla\left(\eta c_{m-2}^{1}, \eta c_{m-1}^{1}, \omega\right), \nabla\left(\eta c_{m-2}^{2}, \eta c_{m-1}^{2}, \omega\right)\right\}\right),
\end{aligned}
$$

with the same manner, we have

$$
\begin{aligned}
\nabla\left(\eta\left(c_{m-1}^{2}\right), \eta\left(c_{m}^{2}\right), \omega\right) & =\nabla\left(\Lambda\left(c_{m-2}^{2}, c_{m-2}^{1}\right), \Lambda\left(c_{m-1}^{2}, c_{m-1}^{1}\right), \omega\right) \\
& \succeq \varphi\left(\min \left\{\nabla\left(\eta c_{m-2}^{2}, \eta c_{m-1}^{2}, \omega\right), \nabla\left(\eta c_{m-2}^{1}, \eta c_{m-1}^{1}, \omega\right)\right\}\right) .
\end{aligned}
$$

This yields

$$
\begin{aligned}
\Omega_{m} & =\min \left\{\nabla\left(\eta\left(c_{m-1}^{1}\right), \eta\left(c_{m}^{1}\right), \omega\right), \nabla\left(\eta\left(c_{m-1}^{2}\right), \eta\left(c_{m}^{2}\right), \omega\right)\right\} \\
& \succeq \varphi\left(\min \left\{\nabla\left(\eta c_{m-2}^{1}, \eta c_{m-1}^{1}, \omega\right), \nabla\left(\eta c_{m-2}^{2}, \eta c_{m-1}^{2}, \omega\right)\right\}\right) \\
& =\varphi\left(\Omega_{m-1}\right) .
\end{aligned}
$$

Thus, we have

$$
v \succeq\left\{\Omega_{m}\right\} \succeq \varphi\left(\Omega_{m-1}\right) \succ\left\{\Omega_{m-1}\right\} \succeq \vartheta .
$$

Thus, $\left\{\theta_{m}(\omega)\right\}$ is a monotonic sequence in $A$. By (3.14) and Remark 2.7, there is $v^{*} \in A$ such that

$$
\lim _{m \rightarrow \infty}\left\{\Omega_{m}\right\}=v^{*} .
$$

Inequality (3.13) suggests that

$$
\begin{aligned}
\Omega_{m} & \succeq \varphi\left(\min \left\{\nabla\left(\eta c_{m-2}^{1}, \eta c_{m-1}^{1}, \omega\right), \nabla\left(\eta c_{m-2}^{2}, \eta c_{m-1}^{2}, \omega\right)\right\}\right), \\
& \geq \varphi\left(\min \left\{\nabla\left(\eta c_{m-2}^{1}, \eta c_{m-1}^{1}, \omega\right), \nabla\left(\eta c_{m-2}^{2}, \eta c_{m-1}^{2}, \omega\right)\right\}\right) \\
& \succ \min \left\{\nabla\left(\eta c_{m-2}^{1}, \eta c_{m-1}^{1}, \omega\right), \nabla\left(\eta c_{m-2}^{2}, \eta c_{m-1}^{2}, \omega\right)\right\} \\
& \geq \nabla\left(\eta c_{m-2}^{1}, \eta c_{m-1}^{1}, \omega\right) .
\end{aligned}
$$

Taking the limit as $m \rightarrow \infty$ in the above inequality and using $\lim _{m \rightarrow \infty} \nabla\left(c_{m}^{1}, c_{m-1}^{1}, \omega\right)=v$, we have

$$
v^{*} \succeq v
$$

Remark 2.8 leads to $v=v^{*}$. Thus

$$
\lim _{m \rightarrow \infty}\left\{\Omega_{m}\right\}=v
$$


We show that $\left\{\eta c_{m}^{1}\right\}$ and $\left\{\eta c_{m}^{2}\right\}$ are Cauchy sequences in $\eta(\partial)$ for $m \in \mathbb{N}$. First we prove that, for each $v$, there are two numbers $n, m \in \mathbb{N}$ such that

$$
\min \left\{\nabla\left(\eta c_{m}^{1}, \eta c_{n}^{1}, \omega\right), \nabla\left(\eta c_{m}^{2}, \eta c_{n}^{2}, \omega\right)\right\} \succ v
$$

Assume the contrary. Thus, for each $v$ and integer $\sigma$, there exist integers $n(\sigma)$ and $m(\sigma)$ with $m(\sigma)>n(\sigma) \geq \sigma$ so that

$$
\min \left\{\nabla\left(\eta c_{m(\sigma)}^{1}, \eta c_{n(\sigma)}^{1}, \omega\right), \nabla\left(\eta c_{m(\sigma)}^{2}, \eta c_{n(\sigma)}^{2}, \omega\right)\right\} \preceq \nu
$$

Consider

$$
\min \left\{\nabla\left(\eta c_{m(\sigma)}^{1}, \eta c_{n(\sigma)-1}^{1}, \omega\right), \nabla\left(\eta c_{m(\sigma)}^{2}, \eta c_{n(\sigma)-1}^{2}, \omega\right)\right\} \succ v
$$

Assume that $n(\sigma)$ the smallest number exceeding $m(\sigma)$ so that (3.15) holds. Let

$$
\pi_{\sigma}(\omega)=\min \left\{\nabla\left(\eta c_{m(\sigma)}^{1}, \eta c_{n(\sigma)}^{1}, \omega\right), \nabla\left(\eta c_{m(\sigma)}^{2}, \eta c_{n(\sigma)}^{2}, \omega\right)\right\} .
$$

By (3.10), and using the fact for each $z_{1}, z_{2}, z_{3}, z_{4} \in \Xi, z_{1} \star z_{2} \succeq \min \left\{z_{1}, z_{3}\right\} \star \min \left\{z_{2}, z_{4}\right\}$, we can write

$$
\begin{aligned}
v \succeq & \pi_{\sigma}(\omega) \succeq \min \left\{\begin{array}{l}
\nabla\left(\eta c_{m(\sigma)}^{1}, \eta c_{n(\sigma)-1}^{1}, \frac{\omega}{2}\right) \star \nabla\left(\eta c_{n(\sigma)-1}^{1}, \eta c_{n(\sigma)}^{1} \frac{\omega}{2}\right), \\
\nabla\left(\eta c_{m(\sigma)}^{2}, \eta c_{n(\sigma)-1}^{2}, \frac{\omega}{2}\right) \star \nabla\left(\eta c_{n(\sigma)-1}^{2}, \eta c_{n(\sigma)}^{2}, \frac{\omega}{2}\right)
\end{array}\right\} \\
\succeq & \min \left\{\min \left\{\nabla\left(\eta c_{m(\sigma)}^{1}, \eta c_{n(\sigma)-1}^{1}, \omega\right), \nabla\left(\eta c_{m(\sigma)}^{2}, \eta c_{n(\sigma)-1}^{2}, \omega\right)\right\}\right. \\
& \star \min \left\{\nabla\left(\eta c_{n(\sigma)-1}^{1}, \eta c_{n(\sigma)}^{1} \frac{\omega}{2}\right), \nabla\left(\eta c_{n(\sigma)-1}^{2}, \eta c_{n(\sigma)}^{2}, \frac{\omega}{2}\right)\right\}, \\
& \min \left\{\nabla\left(\eta c_{m(\sigma)}^{1}, \eta c_{n(\sigma)-1}^{1}, \omega\right), \nabla\left(\eta c_{m(\sigma)}^{2}, \eta c_{n(\sigma)-1}^{2}, \omega\right)\right\} \\
& \left.\star \min \left\{\nabla\left(\eta c_{n(\sigma)-1}^{1}, \eta c_{n(\sigma)}^{1} \frac{\omega}{2}\right), \nabla\left(\eta c_{n(\sigma)-1}^{2}, \eta c_{n(\sigma)}^{2}, \frac{\omega}{2}\right)\right\}\right\} . \\
\succeq & \min \left\{\nabla\left(\eta c_{m(\sigma)}^{1}, \eta c_{n(\sigma)-1}^{1}, \omega\right), \nabla\left(\eta c_{m(\sigma)}^{2}, \eta c_{n(\sigma)-1}^{2}, \omega\right)\right\} \star \theta_{n}\left(\frac{\omega}{2}\right) .
\end{aligned}
$$

Taking $\sigma \rightarrow \infty$ in the above inequality, we get

$$
v \succeq \lim _{\sigma \rightarrow \infty} \pi_{\sigma}(\omega) \succeq v \star \Omega_{n(\sigma)}\left(\frac{\omega}{2}\right)=v \star v=v .
$$

Thus,

$$
\lim _{\sigma \rightarrow \infty} \pi_{\sigma}(\omega)=v, \quad \forall \omega \in A_{\vartheta} .
$$

On the other hand, we get

$$
\begin{aligned}
& \pi_{\sigma}(\omega) \\
& \quad \succeq \min \left\{\begin{array}{l}
\nabla\left(\eta c_{m(\sigma)}^{1}, \eta c_{m(\sigma)+1}^{1}, \frac{\omega}{3}\right) \star \nabla\left(\eta c_{m(\sigma)+1}^{1}, \eta c_{n(\sigma)+1}^{1}, \frac{\omega}{3}\right) \star \nabla\left(\eta c_{n(\sigma)+1}^{1}, \eta c_{n(\sigma)}^{1}, \frac{\omega}{3}\right), \\
\nabla\left(\eta c_{m(\sigma)}^{2}, \eta c_{m(\sigma)+1}^{2}, \frac{\omega}{3}\right) \star \nabla\left(\eta c_{m(\sigma)+1}^{2}, \eta c_{n(\sigma)+1}^{2}, \frac{\omega}{3}\right) \star \nabla\left(\eta c_{n(\sigma)+1}^{2}, \eta c_{n(\sigma)}^{2}, \frac{\omega}{3}\right)
\end{array}\right\}
\end{aligned}
$$




$$
\begin{aligned}
& \qquad \min \left\{\begin{array}{c}
\min \left\{\nabla\left(\eta c_{m(\sigma)}^{1}, \eta c_{m(\sigma)+1}^{1}, \frac{\omega}{3}\right), \nabla\left(\eta c_{m(\sigma)}^{2}, \eta c_{m(\sigma)+1}^{2}, \frac{\omega}{3}\right)\right\} \\
\star \\
\min \left\{\nabla\left(\eta c_{m(\sigma)+1}^{1}, \eta c_{n(\sigma)+1}^{1}, \frac{\omega}{3}\right), \nabla\left(\eta c_{m(\sigma)+1}^{2}, \eta c_{n(\sigma)+1}^{2}, \frac{\omega}{3}\right)\right\} \\
\star \min \left\{\nabla\left(\eta c_{n(\sigma)+1}^{1}, \eta c_{n(\sigma)}^{1}, \frac{\omega}{3}\right), \nabla\left(\eta c_{n(\sigma)+1}^{2}, \eta c_{n(\sigma)}^{2}, \frac{\omega}{3}\right)\right\}, \\
\min \left\{\nabla\left(\eta c_{m(\sigma)}^{1}, \eta c_{m(\sigma)+1}^{1}, \frac{\omega}{3}\right), \nabla\left(\eta c_{m(\sigma)}^{2}, \eta c_{m(\sigma)+1}^{2}, \frac{\omega}{3}\right)\right\} \\
\star \min \left\{\nabla\left(\eta c_{m(\sigma)+1}^{1}, \eta c_{n(\sigma)+1}^{1}, \frac{\omega}{3}\right), \nabla\left(\eta c_{m(\sigma)+1}^{2}, \eta c_{n(\sigma)+1}^{2}, \frac{\omega}{3}\right)\right\} \\
\star \min \left\{\nabla\left(\eta c_{n(\sigma)+1}^{1}, \eta c_{n(\sigma)}^{1}, \frac{\omega}{3}\right), \nabla\left(\eta c_{n(\sigma)+1}^{2}, \eta c_{n(\sigma)}^{2}, \frac{\omega}{3}\right)\right\}
\end{array}\right\} \\
& =\min \left\{\nabla\left(\eta c_{m(\sigma)}^{1}, \eta c_{m(\sigma)+1}^{1}, \frac{\omega}{3}\right), \nabla\left(\eta c_{m(\sigma)}^{2}, \eta c_{m(\sigma)+1}^{2}, \frac{\omega}{3}\right)\right\} \\
& \star \min \left\{\nabla\left(\eta c_{m(\sigma)+1}^{1}, \eta c_{n(\sigma)+1}^{1}, \frac{\omega}{3}\right), \nabla\left(\eta c_{m(\sigma)+1}^{2}, \eta c_{n(\sigma)+1}^{2}, \frac{\omega}{3}\right)\right\} \\
& \star \min \left\{\nabla\left(\eta c_{n(\sigma)+1}^{1}, \eta c_{n(\sigma)}^{1}, \frac{\omega}{3}\right), \nabla\left(\eta c_{n(\sigma)+1}^{2}, \eta c_{n(\sigma)}^{2}, \frac{\omega}{3}\right)\right\} \\
& =\theta_{m(\sigma)}\left(\frac{\omega}{3}\right) \star \min \left\{\nabla\left(\eta c_{m(\sigma)+1}^{1}, \eta c_{n(\sigma)+1}^{1}, \frac{\omega}{3}\right), \nabla\left(\eta c_{m(\sigma)+1}^{2}, \eta c_{n(\sigma)+1}^{2}, \frac{\omega}{3}\right)\right\} \\
& \star \theta_{n(\sigma)}\left(\frac{\omega}{3}\right) .
\end{aligned}
$$

By (3.12), we have

$$
\begin{aligned}
\pi_{\sigma}(\omega) \succeq & \Omega_{m(\sigma)}\left(\frac{\omega}{3}\right) \star \min \left\{\begin{array}{l}
\nabla\left(\Lambda\left(c_{m(\sigma)}^{1}, c_{m(\sigma)}^{2}\right), \Lambda\left(c_{n(\sigma)}^{1}, c_{n(\sigma)}^{2}\right), \frac{\omega}{3}\right), \\
\nabla\left(\Lambda\left(c_{m(\sigma)}^{2}, c_{m(\sigma)}^{1}\right), \Lambda\left(c_{n(\sigma)}^{2}, c_{n(\sigma)}^{1}\right), \frac{\omega}{3}\right)
\end{array}\right\} \star \Omega_{n(\sigma)}\left(\frac{\omega}{3}\right) . \\
\succeq & \Omega_{m(\sigma)}\left(\frac{\omega}{3}\right) \star \min \left\{\varphi\left(\nabla\left(\eta c_{m(\sigma)}^{1}, \eta c_{n(\sigma)}^{1}, \frac{\omega}{3}\right), \nabla\left(\eta c_{m(\sigma)}^{2}, \eta c_{n(\sigma)}^{2}, \frac{\omega}{3}\right)\right)\right\} \\
& \star \Omega_{n(\sigma)}\left(\frac{\omega}{3}\right) \\
= & \Omega_{m(\sigma)}\left(\frac{\omega}{3}\right) \star \varphi\left(\pi_{\sigma}(\omega)\right) \star \Omega_{n(\sigma)}\left(\frac{\omega}{3}\right) .
\end{aligned}
$$

Applying the limit as $\sigma \rightarrow \infty$ in (3.16), and by Definition 2.2, we can write

$$
v \succeq v \star \varphi(v) \star v \succ v \star v \star v=v,
$$

which is a contradiction. Thus $\left\{\eta c_{m}^{1}\right\}$ and $\left\{\eta c_{m}^{2}\right\}$ are Cauchy sequences in $\eta(\partial)$. The completeness of $\eta(\partial)$ and Lemma 2.9 leads to the existence of two elements $c^{1}, c^{2} \in \partial$ so that

$$
\lim _{a \rightarrow \infty} \nabla\left(\eta c_{m}^{1}, c^{1}, \omega\right)=v \quad \text { and } \quad \lim _{a \rightarrow \infty} \nabla\left(\eta c_{m}^{2}, c^{2}, \omega\right)=v \quad \text { for each } \omega \in A_{0}
$$

Also, the continuity of $\eta$ implies that $\left\{\eta \eta c_{m}^{1}\right\}$ and $\left\{\eta \eta c_{m}^{2}\right\}$ are convergent to $\eta c^{1}$ and $\eta c^{2}$ respectively. Moreover, since $\Lambda$ and $\eta$ commute, then we can write

$$
\eta \eta c_{m+1}^{1}=\eta\left(\Lambda\left(c_{m}^{1}, c_{m}^{2}\right)\right)=\Lambda\left(\eta c_{m}^{1}, \eta c_{m}^{2}\right)
$$

and

$$
\eta \eta c_{m+1}^{2}=\eta\left(\Lambda\left(c_{m}^{2}, c_{m}^{1}\right)\right)=\Lambda\left(\eta c_{m}^{2}, \eta c_{m}^{1}\right) .
$$


Thus, by (3.12), we have

$$
\begin{aligned}
\nabla\left(\eta \eta c_{m+1}^{1}, \Lambda\left(c^{1}, c^{2}\right), \omega\right) & =\nabla\left(\Lambda\left(\eta c_{m}^{1}, \eta c_{m}^{2}\right), \Lambda\left(c^{1}, c^{2}\right), \omega\right) \\
& \succeq \varphi\left(\min \left\{\nabla\left(\eta \eta c_{m}^{1}, \eta c^{1}, \omega\right), \nabla\left(\eta \eta c_{m}^{2}, \eta c^{2}, \omega\right)\right\}\right)
\end{aligned}
$$

Passing $m \rightarrow \infty$ in the above inequality, we get

$$
\nabla\left(\eta c^{1}, \Lambda\left(c^{1}, c^{2}\right), \omega\right)=\nu
$$

Hence $\eta c^{1}=\Lambda\left(c^{1}, c^{2}\right)$, similarly, one can prove that $\eta c^{2}=\Lambda\left(c^{2}, c^{1}\right)$. Thus the pair $\left(c^{1}, c^{2}\right)$ is a coupled FP of the mappings $\eta$ and $\Lambda$. Again, by (3.12), we get

$$
\begin{aligned}
\nabla\left(c^{1}, \eta c^{1}, \omega\right) & =\lim _{m \rightarrow \infty}\left(\nabla\left(\eta c_{m+1}^{1}, \eta c^{1}, \omega\right)\right) \\
& =\lim _{m \rightarrow \infty}\left(\nabla\left(\Lambda\left(c_{m}^{1}, c_{m}^{2}\right), \Lambda\left(c^{1}, c^{2}\right), \omega\right)\right) \\
& \succeq \lim _{m \rightarrow \infty}\left(\varphi\left(\min \left\{\nabla\left(\eta c_{m}^{1}, \eta c^{1}, \omega\right), \nabla\left(\eta c_{m}^{2}, \eta c^{2}, \omega\right)\right\}\right)\right) \\
& =\varphi\left(\min \left\{\nabla\left(c^{1}, \eta c^{1}, \omega\right), \nabla\left(c^{2}, \eta c^{2}, \omega\right)\right\}\right) \\
& \succ \min \left\{\nabla\left(c^{1}, \eta c^{1}, \omega\right), \nabla\left(c^{2}, \eta c^{2}, \omega\right)\right\},
\end{aligned}
$$

this implies that

$$
\nabla\left(c^{1}, \eta c^{1}, \omega\right) \succ \min \left\{\nabla\left(c^{1}, \eta c^{1}, \omega\right), \nabla\left(c^{2}, \eta c^{2}, \omega\right)\right\}
$$

similarly, one can write

$$
\nabla\left(c^{2}, \eta c^{2}, \omega\right) \succ \min \left\{\nabla\left(c^{2}, \eta c^{2}, \omega\right), \nabla\left(c^{1}, \eta c^{1}, \omega\right)\right\}
$$

Thus

$$
\begin{aligned}
\min \left\{\nabla\left(c^{1}, \eta c^{1}, \omega\right), \nabla\left(c^{2}, \eta c^{2}, \omega\right)\right\} & \succ \min \left\{\begin{array}{c}
\min \left\{\nabla\left(c^{1}, \eta c^{1}, \omega\right), \nabla\left(c^{2}, \eta c^{2}, \omega\right)\right\} \\
\min \left\{\nabla\left(c^{2}, \eta c^{2}, \omega\right), \nabla\left(c^{1}, \eta c^{1}, \omega\right)\right\}
\end{array}\right\} \\
& =\min \left\{\nabla\left(c^{1}, \eta c^{1}, \omega\right), \nabla\left(c^{2}, \eta c^{2}, \omega\right)\right\} .
\end{aligned}
$$

The last inequality holds only if $\nabla\left(c^{1}, \eta c^{1}, \omega\right)=v$ and $\nabla\left(c^{2}, \eta c^{2}, \omega\right)=v$. Hence $c^{1}=\eta c^{1}$ and $c^{2}=\eta c^{2}$ or, equivalently, $c^{1}=\eta c^{1}=\Lambda\left(c^{1}, c^{2}\right)$ and $c^{2}=\eta c^{2}=\Lambda\left(c^{2}, c^{1}\right)$.

For the uniqueness, assume that $c^{* 1} \in \partial$ with $c^{* 1} \neq c^{1}$ so that

$$
c^{* 1}=\eta c^{* 1}=\Lambda\left(c^{* 1}, c^{* 2}\right) .
$$

Then, by (3.12), we have

$$
\begin{aligned}
\nabla\left(c^{1}, c^{* 1}, \omega\right) & =\nabla\left(\Lambda\left(c^{1}, c^{2}\right), \Lambda\left(c^{* 1}, c^{* 2}\right), \omega\right) \\
& \succeq \varphi\left(\min \left\{\nabla\left(\eta c^{1}, \eta c^{* 1}, \omega\right), \nabla\left(\eta c^{2}, \eta c^{* 2}, \omega\right)\right\}\right)
\end{aligned}
$$




$$
\begin{aligned}
& \succ \min \left\{\nabla\left(c^{1}, c^{* 1}, \omega\right), \nabla\left(c^{2}, c^{* 2}, \omega\right)\right\} \\
& \succeq \nabla\left(c^{1}, c^{* 1}, \omega\right),
\end{aligned}
$$

which is a contradiction. Thus $c^{1}=c^{* 1}$, similarly, one can prove that $c^{2}=c^{* 2}$. Hence $\Lambda$ and $\eta$ have a unique CCFP.

Corollary 3.3 Let $(\partial, \nabla, \star)$ be a complete CVFMS and $\Lambda: \partial \times \partial \rightarrow \partial, \eta: \partial \rightarrow \circlearrowright$ be selfmappings so that

$$
\nabla\left(\Lambda\left(c^{1}, c^{2}\right), \Lambda\left(b^{1}, b^{2}\right), \omega\right) \succeq \sqrt{\min \left\{\nabla\left(\eta c^{1}, \eta b^{1}, \omega\right), \nabla\left(\eta c^{2}, \eta b^{2}, \omega\right)\right\}}
$$

for all $c^{1}, c^{2}, b^{1}, b^{2} \in \partial, \omega \in A_{\vartheta}$. Assume that $\Lambda$ and $\eta$ satisfy the same stipulations of Theorem 3.1 with $\varphi \in \Phi$. Then $\Lambda$ and $\eta$ have a unique (CCFP), whenever $\lim _{m \rightarrow \infty} \nabla\left(c_{m}^{1}, c_{m-1}^{1}\right.$, $\omega)=v$.

Corollary 3.4 Let $(\partial, \nabla, \star)$ be a complete CVFMS and $\Lambda: \partial \times \partial \rightarrow \partial, \eta: \partial \rightarrow \partial$ be selfmappings so that

$$
\begin{aligned}
\nabla\left(\Lambda\left(c^{1}, c^{2}\right), \Lambda\left(b^{1}, b^{2}\right), \omega\right) \succeq & 2 \min \left\{\nabla\left(\eta c^{1}, \eta b^{1}, \omega\right), \nabla\left(\eta c^{2}, \eta b^{2}, \omega\right)\right\} \\
& -\left(\min \left\{\nabla\left(\eta c^{1}, \eta b^{1}, \omega\right), \nabla\left(\eta c^{2}, \eta b^{2}, \omega\right)\right\}\right)^{2}
\end{aligned}
$$

for all $c^{1}, c^{2}, b^{1}, b^{2} \in \partial, \omega \in A_{\vartheta}$. Assume that $\Lambda$ and $\eta$ satisfy the same stipulations of Theorem 3.1 with $\varphi \in \Phi$. Then $\Lambda$ and $\eta$ have a unique CCFP, whenever $\lim _{m \rightarrow \infty} \nabla\left(c_{m}^{1}, c_{m-1}^{1}\right.$, $\omega)=v$.

Examples below support our theoretical results.

Example 3.1 Let $\partial=\{0\} \cup\left\{\frac{1}{m}: m \in \mathbb{N}\right\}$ with the metric $\Theta$ defined by

$$
\Theta\left(c^{1}, c^{2}\right)=\left|c^{1}-c^{2}\right|, \quad \forall c^{1}, c^{2} \in \partial .
$$

Define a $t$-norm “ $\star$ " by $p \star q=\min \{p, q\}, p, q \in \Xi$. Assume that $\nabla$ is the complex-valued fuzzy set defined by

$$
\nabla\left(c^{1}, c^{2}, \omega\right)=\frac{\omega}{\omega+\Theta\left(c^{1}, c^{2}\right)} v
$$

for $\omega \in A_{0}$. It is obvious that $(\partial, \nabla, \star)$ is a CVFMS. Define two mappings $\Lambda: \partial \times \partial \rightarrow \partial$ and $\eta: \partial \rightarrow \partial$ by

$$
\Lambda\left(c^{1}, c^{2}\right)=\frac{c^{1}+c^{2}}{8} \text { and } \eta c^{1}=\frac{c^{1}}{4}
$$


It is evident that $\Lambda(\partial \times \partial) \subseteq \eta(\partial)$ and $\eta$ is continuous. Since

$$
\begin{aligned}
\nabla\left(\Lambda\left(c^{1}, c^{2}\right), \Lambda\left(b^{1}, b^{2}\right), \omega\right) & =\nabla\left(\frac{c^{1}+c^{2}}{8}, \frac{b^{1}+b^{2}}{8}, \omega\right) \\
& =\frac{\omega v}{\omega+\left|\frac{c^{1}+c^{2}}{8}-\frac{b^{1}+b^{2}}{8}\right|} \\
& =\frac{\omega v}{\omega+\left|\frac{c^{1}-b^{1}}{8}+\frac{c^{2}-b^{2}}{8}\right|} \\
& \succeq \frac{\omega v}{\omega+\left|\frac{c^{1}-b^{1}}{8}\right|}+\frac{\omega}{\omega+\left|\frac{c^{2}-b^{2}}{8}\right|} v \\
& \succeq \frac{\omega v}{\omega+\left|\frac{c^{1}-b^{1}}{4}\right|}+\frac{\omega}{\omega+\left|\frac{c^{1}-b^{1}}{4}\right|} v \\
& \succeq \max \left\{\frac{\omega}{\omega+\left|\frac{c^{1}-b^{1}}{4}\right|} v, \frac{\omega}{\omega+\mid \frac{c^{2}-b^{2}}{4}} v\right\} \\
& =\max \left\{\nabla\left(\eta c^{1}, \eta b^{1}, \omega\right), \nabla\left(\eta c^{2}, \eta b^{2}, \omega\right)\right\} .
\end{aligned}
$$

Then we can write

$$
\left(v-\nabla\left(\Lambda\left(c^{1}, c^{2}\right), \Lambda\left(b^{1}, b^{2}\right), \omega\right)\right) \preceq\left(v-\max \left\{\nabla\left(\eta c^{1}, \eta b^{1}, \omega\right), \nabla\left(\eta c^{2}, \eta b^{2}, \omega\right)\right\}\right) .
$$

Thus, all the requirements of Theorem 3.1 hold with $\mu=1$ and $(0,0)$ is a unique coupled FP.

Example 3.2 Assume that all hypotheses of Example 3.1 hold. Let $\nabla$ be the complexvalued fuzzy set defined by

$$
\nabla\left(c^{1}, c^{2}, \omega\right)=v e^{\frac{\left|c^{1}-c^{2}\right|}{\omega}}
$$

for $\omega \in A_{0}$. It is obvious that $(\partial, \nabla, \star)$ is a CVFMS. Define two mappings $\Lambda: \partial \times \partial \rightarrow \partial$ and $\eta: \partial \rightarrow \partial$ by

$$
\Lambda\left(c^{1}, c^{2}\right)=\frac{2 c^{1}+c^{2}}{32 \sqrt{17}} \quad \text { and } \quad \eta c^{1}=\frac{c^{1}}{4}
$$

and $\varphi(l)=\sqrt{l}, l>0$. It is evident that $\Lambda(\partial \times \partial) \subseteq \eta(\partial)$ and $\eta$ is continuous. Since

$$
\begin{aligned}
\left|\frac{2 c^{1}-2 b^{1}}{32 \sqrt{17}}+\frac{c^{2}-b^{2}}{32 \sqrt{17}}\right| & \preceq \frac{2}{32}\left(\left|\frac{c^{1}}{4}-\frac{b^{1}}{4}\right|+\left|\frac{c^{2}}{4}-\frac{b^{2}}{4}\right|\right) \\
& =\frac{1}{16}\left(\left|\frac{c^{1}}{4}-\frac{b^{1}}{4}\right|+\left|\frac{c^{2}}{4}-\frac{b^{2}}{4}\right|\right) \\
& \leq \frac{1}{8} \max \left\{\left|\frac{c^{1}}{4}-\frac{b^{1}}{4}\right|,\left|\frac{c^{2}}{4}-\frac{b^{2}}{4}\right|\right\},
\end{aligned}
$$


it follows that

$$
\begin{aligned}
\nabla\left(\Lambda\left(c^{1}, c^{2}\right), \Lambda\left(b^{1}, b^{2}\right), \omega\right) & =v e^{\frac{\left|\frac{2 c^{1}}{32 \sqrt{2}}-\frac{2 b^{1}+b^{2}}{32 \sqrt{17}}\right|}{\omega}} \\
& =v e^{\frac{\left|\frac{2 c^{1}-2 b^{1}}{32 \sqrt{17}}+\frac{c^{2}-b^{2}}{32 \sqrt{17}}\right|}{\omega}} \\
& \succeq v e^{\frac{\max || \frac{c^{1}}{4}-\frac{b^{1}}{4}|,| \frac{c^{2}}{4}-\frac{b^{2}}{4}||}{8 \omega}} \\
& \succeq \sqrt{v e^{\frac{\left.\max \left|\frac{c^{1}}{4}-\frac{b^{1}}{4}\right|,\left|\frac{c^{2}}{4}-\frac{b^{2}}{4}\right|\right\}}{4 \omega}}} \\
& \succeq \sqrt{\nu \max \left\{e^{\frac{\mid c^{1}}{4}-\frac{b^{1}}{4} \mid}, e^{\frac{\mid c^{2}}{4}-\frac{b^{2} \mid}{\omega}}\right\}} \\
& \succeq \sqrt{\nu \min \left\{e^{\frac{\mid c^{1}}{4}-\frac{b^{1}}{\omega} \mid}, e^{\frac{\left|c^{2}-\frac{b^{2}}{4}\right|}{\omega}}\right\}} \\
& =\varphi\left(\min \left\{\nabla\left(\eta c^{1}, \eta b^{1}, \omega\right), \nabla\left(\eta c^{2}, \eta b^{2}, \omega\right)\right\}\right)
\end{aligned}
$$

for all $c^{1}, c^{2}, b^{1}, b^{2} \in \partial, \omega \in A_{0}$. Also, for any sequence $\left\{c_{m}^{1}\right\}_{m \in \mathbb{N}}=\frac{1}{m}$, we can write

$$
\lim _{m \rightarrow \infty} \nabla\left(c_{m}^{1}, c_{m-1}^{1}, \omega\right)=v e^{\frac{\left|\frac{1}{m}-\frac{1}{m-1}\right|}{\omega}}=v e^{0}=v
$$

Thus the assumptions of Theorem 3.2 hold and $(0,0)$ is a unique CCFP of the mappings $\Lambda$ and $\eta$.

\section{Existence solution for a coupled system of impulsive fractional differential equations}

In this section, we study the existence and uniqueness of a coupled system of nonlinear fractional differential equation with impulses (1.1)

If necessary, the reader can refer to [36] for a more detailed explanation of the background of the problem. Here we use our derived fixed point theorems to prove the existence of solutions to problem (1.1). Let $\mathcal{X}^{\prime}=\mathrm{X} \times \mathrm{Y}$ with the norms $\|(\mathrm{x}, \mathrm{y})\|=\|\mathrm{x}\|_{0}+\|\mathrm{y}\|_{0}$ and $\|(x, y)\|=\max \left\{\|x\|_{0},\|y\|_{0}\right\}$. Define the operator $\mathcal{T}: \mathcal{X}^{\prime} \rightarrow \mathcal{X}^{\prime}$ by

$$
\mathcal{T}(\mathrm{x}, \mathrm{y})(\mathrm{t})=\left(\mathrm{T}_{1}(\mathrm{x}, \mathrm{y})(\mathrm{t}), \mathrm{T}_{2}(\mathrm{x}, \mathrm{y})(\mathrm{t})\right)^{\mathrm{T}}, \quad \forall(\mathrm{x}, \mathrm{y}) \in \mathcal{X}^{\prime}, \mathrm{t} \in[0,1]
$$

where

$$
\mathcal{T}_{1}(\mathrm{x}, \mathrm{y})(\mathrm{t})=\left\{\begin{aligned}
&-\frac{1}{\Gamma(\mu)} \int_{0}^{\mathrm{t}}(\mathrm{t}-\mathrm{s}) \mathrm{h}\left(\mathrm{s},{ }^{\mathrm{c}} \mathrm{D}^{\varrho} \mathrm{x}(\mathrm{s}),{ }^{\mathrm{c}} \mathrm{D}^{\rho} \mathrm{y}(\mathrm{s})\right) \mathrm{ds}+\mathrm{e}^{*} \mathrm{t}^{2}, \quad \mathrm{t} \in\left[0, \mathrm{t}_{1}\right] \\
&-\frac{1}{\Gamma(\mu)} \int_{\mathrm{t}_{\mathrm{m}}}^{\mathrm{t}}(\mathrm{t}-\mathrm{s})^{\mu-1} \mathrm{~h}\left(\mathrm{~s}^{\mathrm{c}} \mathrm{D}^{\varrho} \mathrm{x}(\mathrm{s}),{ }^{\mathrm{c}} \mathrm{D}^{\rho} \mathrm{y}(\mathrm{s})\right) \mathrm{ds} \\
& \quad-\frac{1}{\Gamma(\mu)} \sum_{\mathrm{j}=1}^{\mathrm{m}} \int_{\mathrm{t}_{\mathrm{j}-1}}^{\mathrm{t}_{\mathrm{j}}}\left(\mathrm{t}_{\mathrm{j}}-\mathrm{s}\right)^{\mu-1} \mathrm{~h}\left(\mathrm{~s},{ }^{\mathrm{c}} \mathrm{D}^{\varrho} \mathrm{x}(\mathrm{s}),{ }^{\mathrm{c}} \mathrm{D}^{\rho} \mathrm{y}(\mathrm{s})\right) \mathrm{ds} \\
& \quad-\frac{1}{\Gamma(\mu-1)} \sum_{\mathrm{j}=1}^{\mathrm{m}}\left(\mathrm{t}-\mathrm{t}_{\mathrm{j}}\right) \int_{\mathrm{t}_{\mathrm{j}-1}}^{\mathrm{t}_{\mathrm{j}}}\left(\mathrm{t}_{\mathrm{j}}-\mathrm{s}\right)^{\mu-2} \mathrm{~h}\left(\mathrm{~s},{ }^{\mathrm{c}} \mathrm{D}^{\varrho} \mathrm{x}(\mathrm{s}),{ }^{\mathrm{c}} \mathrm{D}^{\rho} \mathrm{y}(\mathrm{s})\right) \mathrm{ds} \\
&-\frac{1}{2 \Gamma(\mu-2)} \sum_{\mathrm{j}=1}^{\mathrm{m}}\left(\mathrm{t}-\mathrm{t}_{\mathrm{j}}\right)^{2} \int_{\mathrm{t}_{\mathrm{j}-1}}^{\mathrm{t}_{\mathrm{j}}}\left(\mathrm{t}_{\mathrm{j}}-\mathrm{s}\right)^{\mu-3} \mathrm{~h}\left(\mathrm{~s},{ }^{\mathrm{c}} \mathrm{D}^{\varrho} \mathrm{x}(\mathrm{s}),{ }^{\mathrm{c}} \mathrm{D}^{\rho} \mathrm{y}(\mathrm{s})\right) \mathrm{ds} \\
&+\sum_{\mathrm{j}=1}^{\mathrm{m}} \mathcal{M}_{1 \mathrm{j}}\left(\mathrm{x}\left(\mathrm{t}_{\mathrm{j}}\right)\right)+\sum_{\mathrm{j}=1}^{\mathrm{m}}\left(\mathrm{t}-\mathrm{t}_{\mathrm{j}}\right) \mathcal{N}_{1 \mathrm{j}}\left(\mathrm{x}\left(\mathrm{t}_{\mathrm{j}}\right)\right) \\
&+\sum_{\mathrm{j}=1}^{\mathrm{m}} \frac{\left(\mathrm{t}-\mathrm{t}_{\mathrm{j}}\right)^{2}}{2} \mathcal{O}_{1 \mathrm{j}}\left(\mathrm{x}\left(\mathrm{t}_{\mathrm{j}}\right)\right)+\mathrm{c}^{*} \mathrm{t}^{2}, \\
& \mathrm{t}\left(\mathrm{t}_{\mathrm{m}}, \mathrm{t}_{\mathrm{m}+1}\right], 1 \leq \mathrm{m} \leq \mathrm{n},
\end{aligned}\right.
$$


and

$$
\mathcal{T}_{2}(\mathrm{x}, \mathrm{y})(\mathrm{t})=\left\{\begin{array}{c}
-\frac{1}{\Gamma(\gamma)} \int_{0}^{\mathrm{t}}(\mathrm{t}-\mathrm{s})^{\gamma-1} \mathrm{~g}\left(\mathrm{~s},{ }^{\mathrm{c}} \mathrm{D}^{\varrho} \mathrm{x}(\mathrm{s}),{ }^{\mathrm{c}} \mathrm{D}^{\rho} \mathrm{y}(\mathrm{s})\right) \mathrm{ds}+\mathrm{e}^{*} \mathrm{t}^{2}, \quad \mathrm{t} \in\left[0, \mathrm{t}_{1}\right], \\
-\frac{1}{\Gamma(\gamma)} \int_{\mathrm{t}_{\mathrm{m}}}^{\mathrm{t}}(\mathrm{t}-\mathrm{s})^{\gamma-1} \mathrm{~g}\left(\mathrm{~s},{ }^{\mathrm{c}} \mathrm{D}^{\varrho} \mathrm{x}(\mathrm{s}),{ }^{\mathrm{c}} \mathrm{D}^{\rho} \mathrm{y}(\mathrm{s})\right) \mathrm{ds} \\
\quad-\frac{1}{\Gamma(\gamma)} \sum_{\mathrm{j}=1}^{\mathrm{m}} \int_{\mathrm{t}_{\mathrm{j}-1}}^{\mathrm{t}_{\mathrm{j}}}\left(\mathrm{t}_{\mathrm{j}}-\mathrm{s}\right)^{\gamma-1} \mathrm{~g}\left(\mathrm{~s},{ }^{\mathrm{c}} \mathrm{D}^{\varrho} \mathrm{x}(\mathrm{s}),{ }^{\mathrm{c}} \mathrm{D}^{\rho} \mathrm{y}(\mathrm{s})\right) \mathrm{ds} \\
\quad-\frac{1}{\Gamma(\gamma-1)} \sum_{\mathrm{j}=1}^{\mathrm{m}}\left(\mathrm{t}-\mathrm{t}_{\mathrm{j}}\right) \int_{\mathrm{t}_{\mathrm{j}-1}}^{\mathrm{t}_{\mathrm{j}}}\left(\mathrm{t}_{\mathrm{j}}-\mathrm{s}\right)^{\gamma-2} \mathrm{~g}\left(\mathrm{~s},{ }^{\mathrm{c}} \mathrm{D}^{\varrho} \mathrm{x}(\mathrm{s}),{ }^{\mathrm{c}} \mathrm{D}^{\rho} \mathrm{y}(\mathrm{s})\right) \mathrm{ds} \\
\quad-\frac{1}{2 \Gamma(\gamma-2)} \sum_{\mathrm{j}=1}^{\mathrm{m}}\left(\mathrm{t}-\mathrm{t}_{\mathrm{j}}\right)^{2} \int_{\mathrm{t}_{\mathrm{j}-1}}^{\mathrm{t}_{\mathrm{j}}}\left(\mathrm{t}_{\mathrm{j}}-\mathrm{s}\right)^{\gamma-3} \mathrm{~g}\left(\mathrm{~s},{ }^{\mathrm{c}} \mathrm{D}^{\varrho} \mathrm{x}(\mathrm{s}),{ }^{\mathrm{c}} \mathrm{D}^{\rho} \mathrm{y}(\mathrm{s})\right) \mathrm{ds} \\
\quad+\sum_{\mathrm{j}=1}^{\mathrm{m}} \mathcal{M}_{2 \mathrm{j}}\left(\mathrm{x}\left(\mathrm{t}_{\mathrm{j}}\right)\right)+\sum_{\mathrm{j}=1}^{\mathrm{m}}\left(\mathrm{t}-\mathrm{t}_{\mathrm{j}}\right) \mathcal{N}_{2 \mathrm{j}}\left(\mathrm{x}\left(\mathrm{t}_{\mathrm{j}}\right)\right) \\
\quad+\sum_{\mathrm{j}=1}^{\mathrm{m}} \frac{\left(\mathrm{t}-\mathrm{t}_{\mathrm{j}}\right)^{2}}{2} \mathcal{O}_{2 \mathrm{j}}\left(\mathrm{x}\left(\mathrm{t}_{\mathrm{j}}\right)\right)+\mathrm{c}^{*} \mathrm{t}^{2}, \\
\mathrm{t} \in\left(\mathrm{t}_{\mathrm{m}}, \mathrm{t}_{\mathrm{m}+1}\right], 1 \leq \mathrm{m} \leq \mathrm{n},
\end{array}\right.
$$

with

$$
\begin{aligned}
& \mathrm{e}^{*}=\mathcal{Z}_{\mu}\left[-\frac{1}{\Gamma(\mu-\omega)} \int_{\mathrm{t}_{\mathrm{j}}}^{\tau}(\tau-\mathrm{s})^{\mu-\omega-1} \mathrm{~h}\left(\mathrm{~s},{ }^{\mathrm{c}} \mathrm{D}^{\varrho} \mathrm{x}(\mathrm{s}),{ }^{\mathrm{c}} \mathrm{D}^{\rho} \mathrm{y}(\mathrm{s})\right) \mathrm{ds},\right. \\
& -\frac{\tau^{1-\omega}}{\Gamma(\mu-1) \Gamma(2-\omega)} \sum_{\mathrm{j}=1}^{\mathrm{m}} \int_{\mathrm{t}_{\mathrm{j}-1}}^{\mathrm{t}_{\mathrm{j}}}\left(\mathrm{t}_{\mathrm{j}}-\mathrm{s}\right)^{\mu-2} \mathrm{~h}\left(\mathrm{~s},{ }^{\mathrm{c}} \mathrm{D}^{\varrho} \mathrm{x}(\mathrm{s}),{ }^{\mathrm{c}} \mathrm{D}^{\rho} \mathrm{y}(\mathrm{s})\right) \mathrm{ds} \\
& -\frac{\tau^{2-\omega}}{\Gamma(\mu-2) \Gamma(3-\omega)} \sum_{j=1}^{m} \int_{t_{j-1}}^{t_{j}}\left(t_{j}-s\right){ }^{\mu-3} h\left(s,{ }^{c} D^{\varrho} x(s),{ }^{c} D^{\rho} y(s)\right) d s \\
& +\frac{\tau^{1-\omega}}{\Gamma(\mu-1) \Gamma(2-\omega)} \sum_{j=1}^{\mathrm{m}} \mathrm{t}_{\mathrm{j}} \int_{\mathrm{t}_{\mathrm{j}-1}}^{\mathrm{t}_{\mathrm{j}}}\left(\mathrm{t}_{\mathrm{j}}-\mathrm{s}\right)^{\mu-3} \mathrm{~h}\left(\mathrm{~s},{ }^{\mathrm{c}} \mathrm{D}^{\varrho} \mathrm{x}(\mathrm{s}),{ }^{\mathrm{c}} \mathrm{D}^{\rho} \mathrm{y}(\mathrm{s})\right) \mathrm{ds} \\
& +\frac{\tau^{1-\omega}}{\Gamma(2-\omega)} \sum_{j=1}^{m} \mathcal{N}_{1 j}\left(x\left(t_{j}\right)\right)+\frac{\tau^{2-\omega}}{\Gamma(3-\omega)} \sum_{j=1}^{m} \mathcal{O}_{1 j}\left(x\left(t_{j}\right)\right)+\frac{\tau^{1-\omega}}{\Gamma(2-\omega)} \sum_{j=1}^{m} t_{j} \mathcal{O}_{1 j}\left(x\left(t_{j}\right)\right) \\
& +\frac{1}{\Gamma(\mu-2)} \int_{\mathrm{t}_{\mathrm{m}}}^{1}(1-\mathrm{s})^{\mu-3} \mathrm{~h}\left(\mathrm{~s},{ }^{\mathrm{c}} \mathrm{D}^{\varrho} \mathrm{x}(\mathrm{s}),{ }^{\mathrm{c}} \mathrm{D}^{\rho} \mathrm{y}(\mathrm{s})\right) \mathrm{ds} \\
& \left.+\frac{1}{\Gamma(\mu-2)} \sum_{j=1}^{m} \int_{t_{j-1}}^{t_{j}}\left(t_{j}-s\right)^{\mu-3} h\left(s,{ }^{c} D^{\varrho} x(s),{ }^{c} D^{\rho} y(s)\right) d s+\sum_{j=1}^{m} \mathcal{O}_{1 j}\left(x\left(t_{j}\right)\right)\right] \text {, }
\end{aligned}
$$

and

$$
\begin{aligned}
\mathrm{c}^{*}= & \mathcal{Z}_{\gamma}\left[-\frac{1}{\Gamma(\gamma-\xi)} \int_{\mathrm{t}_{\mathrm{j}}}^{\epsilon}(\epsilon-\mathrm{s})^{\gamma-\xi-1} \mathrm{~g}\left(\mathrm{~s},{ }^{\mathrm{c}} \mathrm{D}^{\varrho} \mathrm{x}(\mathrm{s}),{ }^{\mathrm{c}} \mathrm{D}^{\rho} \mathrm{y}(\mathrm{s})\right) \mathrm{ds}\right. \\
& -\frac{\epsilon^{1-\xi}}{\Gamma(\gamma-1) \Gamma(2-\xi)} \sum_{\mathrm{j}=1}^{\mathrm{m}} \int_{\mathrm{t}_{\mathrm{j}-1}}^{\mathrm{t}_{\mathrm{j}}}\left(\mathrm{t}_{\mathrm{j}}-\mathrm{s}\right)^{\gamma-2} \mathrm{~g}\left(\mathrm{~s},{ }^{\mathrm{c}} \mathrm{D}^{\varrho} \mathrm{x}(\mathrm{s}),{ }^{\mathrm{c}} \mathrm{D}^{\rho} \mathrm{y}(\mathrm{s})\right) \mathrm{ds} \\
& -\frac{\epsilon^{2-\xi}}{\Gamma(\gamma-2) \Gamma(3-\xi)} \sum_{\mathrm{j}=1}^{\mathrm{m}} \int_{\mathrm{t}_{\mathrm{j}-1}}^{\mathrm{t}_{\mathrm{j}}}\left(\mathrm{t}_{\mathrm{j}}-\mathrm{s}\right)^{\gamma-3} \mathrm{~g}\left(\mathrm{~s},{ }^{\mathrm{c}} \mathrm{D}^{\varrho} \mathrm{x}(\mathrm{s}),{ }^{\mathrm{c}} \mathrm{D}^{\rho} \mathrm{y}(\mathrm{s})\right) \mathrm{ds} \\
& +\frac{\epsilon^{1-\xi}}{\Gamma(\gamma-1) \Gamma(2-\xi)} \sum_{\mathrm{j}=1}^{\mathrm{m}} \mathrm{t}_{\mathrm{j}} \int_{\mathrm{t}_{\mathrm{j}-1}}^{\mathrm{t}_{\mathrm{j}}}\left(\mathrm{t}_{\mathrm{j}}-\mathrm{s}\right)^{\gamma-3} \mathrm{~g}\left(\mathrm{~s},{ }^{\mathrm{c}} \mathrm{D}^{\varrho} \mathrm{x}(\mathrm{s}),{ }^{\mathrm{c}} \mathrm{D}^{\rho} \mathrm{y}(\mathrm{s})\right) \mathrm{ds}
\end{aligned}
$$




$$
\begin{aligned}
& +\frac{\epsilon^{1-\xi}}{\Gamma(2-\xi)} \sum_{j=1}^{m} \mathcal{N}_{1 j}\left(x\left(t_{j}\right)\right)+\frac{\epsilon^{2-\xi}}{\Gamma(3-\xi)} \sum_{j=1}^{m} \mathcal{O}_{1 j}\left(x\left(t_{j}\right)\right)+\frac{\epsilon^{1-\xi}}{\Gamma(2-\xi)} \sum_{j=1}^{m} t_{j} \mathcal{O}_{1 j}\left(x\left(t_{j}\right)\right) \\
& +\frac{1}{\Gamma(\gamma-2)} \int_{t_{m}}^{1}(1-s)^{\gamma-3} g\left(s,{ }^{c} D^{\varrho} x(s),{ }^{c} D^{\rho} y(s)\right) d s \\
& \left.+\frac{1}{\Gamma(\gamma-2)} \sum_{j=1}^{m} \int_{t_{j-1}}^{t_{j}}\left(t_{j}-s\right)^{\gamma-3} g\left(s,{ }^{c} D^{\varrho} x(s),{ }^{c} D^{\rho} y(s)\right) d s+\sum_{j=1}^{m} \mathcal{O}_{1 j}\left(x\left(t_{j}\right)\right)\right]
\end{aligned}
$$

where $\mathcal{Z}_{\mu}=\frac{\Gamma(3-\omega)}{2 \Gamma(3-\omega)-\tau^{2-\omega}}$ and $\mathcal{Z}_{\gamma}=\frac{\Gamma(3-\xi)}{2 \Gamma(3-\xi)-\tau^{2-\xi}}$. Thus, solving problem (1.1) is equivalent to obtaining a fixed point of the operator $\mathcal{T}$. Next, we have to prove the uniqueness of solutions of problem (1.1).

Theorem 4.1 Let the following conditions $\left(\mathbb{H}_{1}\right)-\left(\mathbb{H}_{4}\right)$ hold, and then the boundary value problem (1.1) has a unique solution.

$\left(\mathbb{H}_{1}\right)$ : For all $\mathrm{t} \in \mathrm{J}$ and $\mathrm{x}_{\mathrm{j}}, \mathrm{y}_{\mathrm{j}} \in \mathbb{R}, \mathrm{j}=1,2$, there exist some positive constants $\mathrm{A}_{\mathrm{j}}, \mathrm{B}_{\mathrm{j}},(\mathrm{j}=1,2$, such that

$$
\begin{aligned}
& \left|h\left(t, x_{1}, y_{1}\right)-h\left(t, x_{2}, y_{2}\right)\right| \leq A_{1}\left|x_{1}-x_{2}\right|+A_{2}\left|y_{1}-y_{2}\right| \\
& \left|g\left(t, x_{1}, y_{1}\right)-g\left(t, x_{2}, y_{2}\right)\right| \leq B_{1}\left|x_{1}-x_{2}\right|+B_{2}\left|y_{1}-y_{2}\right|
\end{aligned}
$$

$\left(\mathbb{H}_{2}\right): \mathrm{x}_{\mathrm{j}}, \mathrm{y}_{\mathrm{j}} \in \mathbb{R}$, there exist some positive constants $\mathrm{L}_{\mathrm{j}}, \hat{\mathrm{L}}_{j \mathrm{~m}}, \check{\mathrm{L}}_{\mathrm{jm}},(\mathrm{j}=1,2 ; \mathrm{m}=1,2, \ldots, \mathrm{n})$ such that

$$
\begin{aligned}
& \left|\mathcal{M}_{j \mathrm{jm}}(\mathrm{x})-\mathcal{M}_{j \mathrm{~m}}(\mathrm{y})\right| \leq \mathrm{L}_{j \mathrm{~m}}|\mathrm{x}-\mathrm{y}| \\
& \left|\mathcal{N}_{j \mathrm{jm}}(\mathrm{x})-\mathcal{N}_{\text {jm }}(\mathrm{y})\right| \leq \hat{\mathrm{L}}_{\mathrm{jm}}|\mathrm{x}-\mathrm{y}| \\
& \left|\mathcal{O}_{j \mathrm{jm}}(\mathrm{x})-\mathcal{O}_{j \mathrm{jm}}(\mathrm{y})\right| \leq \check{\mathrm{L}}_{\mathrm{jm}}|\mathrm{x}-\mathrm{y}|
\end{aligned}
$$

$\left(\mathbb{H}_{3}\right):\left[\left(\mathrm{A}_{1}+\mathrm{A}_{2}\right)\left(\frac{2}{\Gamma(\mu+1)}+\frac{1}{\Gamma(\mu)}+\frac{1}{2 \Gamma(\mu-1)}+\frac{\mathcal{Z}_{\mu}}{\Gamma(\mu-\omega+1)}+\frac{\mathcal{Z}_{\mu} \tau^{1-\omega}}{\Gamma(\mu) \Gamma(2-\omega)}+\frac{\mathcal{Z}_{\mu} \tau^{2-\omega}}{\Gamma(\mu-1) \Gamma(3-\omega)}+\frac{\mathcal{Z}_{\mu} \tau^{1-\omega}}{\Gamma(\mu-1) \Gamma(2-\omega)}+\right.\right.$ $\left.\frac{2 \mathcal{Z}_{\mu}}{\Gamma(\mu-1)}\right)+\sum_{j=1}^{\mathrm{m}} \mathrm{L}_{1 j}+\sum_{\mathrm{j}=1}^{\mathrm{m}} \hat{\mathrm{L}}_{1 \mathrm{j}}+\sum_{\mathrm{j}=1}^{\mathrm{m}} \frac{\mathcal{Z}_{\mu} 1^{1-\omega}}{\Gamma(2-\omega)} \hat{\mathrm{L}}_{1 \mathrm{j}}+\frac{\mathcal{Z}_{\mu} \tau^{2-\omega}}{\Gamma(3-\omega)} \sum_{\mathrm{j}=1}^{\mathrm{m}} \check{\mathrm{L}}_{1 \mathrm{j}}+\frac{\mathcal{Z}_{\mu} \tau^{1-\omega}}{\Gamma(2-\omega)} \sum_{\mathrm{j}=1}^{\mathrm{m}} \check{\mathrm{L}}_{1 \mathrm{j}}+$ $\left.\mathcal{Z}_{\mu} \sum_{\mathrm{j}=1}^{\mathrm{m}} \check{\mathrm{L}}_{1 \mathrm{j}}+\frac{1}{2} \sum_{\mathrm{j}=1}^{\mathrm{m}} \check{\mathrm{L}}_{1 \mathrm{j}}\right]<1$ and $\left[\left(\mathrm{B}_{1}+\mathrm{B}_{2}\right)\left(\frac{2}{\Gamma(\gamma+1)}+\frac{1}{\Gamma(\gamma)}+\frac{1}{2 \Gamma(\gamma-1)}+\frac{\mathcal{Z}_{\gamma}}{\Gamma(\gamma-\xi+1)}+\right.\right.$ $\left.\frac{\mathcal{Z}_{\gamma} \epsilon^{1-\xi}}{\Gamma(\gamma) \Gamma(2-\xi)}+\frac{\mathcal{Z}_{\gamma} \epsilon^{2-\xi}}{\Gamma(\gamma-1) \Gamma(3-\xi)}+\frac{\mathcal{Z}_{\gamma} \epsilon^{1-\xi}}{\Gamma(\gamma-1) \Gamma(2-\xi)}+\frac{2 \mathcal{Z}_{\gamma}}{\Gamma(\gamma-1)}\right)+\sum_{j=1}^{\mathrm{m}} \mathrm{L}_{2 j}+\sum_{\mathrm{j}=1}^{\mathrm{m}} \hat{\mathrm{L}}_{2 \mathrm{j}}+\sum_{\mathrm{j}=1}^{\mathrm{m}} \frac{\mathcal{Z}_{\gamma} \epsilon^{1-\xi}}{\Gamma(2-\xi)} \hat{\mathrm{L}}_{2 \mathrm{j}}+$ $\left.\frac{\mathcal{Z}_{\gamma} \epsilon^{2-\xi}}{\Gamma(3-\xi)} \sum_{\mathrm{j}=1}^{\mathrm{m}} \check{\mathrm{L}}_{2 \mathrm{j}}+\frac{\mathcal{Z}_{\gamma} \epsilon^{1-\xi}}{\Gamma(2-\xi)} \sum_{\mathrm{j}=1}^{\mathrm{m}} \check{\mathrm{L}}_{2 \mathrm{j}}+\mathcal{Z}_{\gamma} \sum_{\mathrm{j}=1}^{\mathrm{m}} \check{\mathrm{L}}_{1 \mathrm{j}}+\frac{1}{2} \sum_{\mathrm{j}=1}^{\mathrm{m}} \check{\mathrm{L}}_{1 \mathrm{j}}\right]<1$,

$\left.\left(\mathbb{H}_{4}\right): \frac{\omega}{\omega+\left\|\left(\eta \mathrm{x}_{1}, \eta \mathrm{x}_{2}\right)\right\|} v=\min \left\{\frac{\omega}{\omega+\left\|\left(\eta \mathrm{x}_{1}, \eta \mathrm{x}_{2}\right)\right\|} v, \frac{\omega}{\omega+\left\|\left(\eta \mathrm{y}_{1}, \eta \mathrm{y}_{2}\right)\right\|} v\right)\right\}$ and

$$
\left\|\left(\mathrm{x}_{1}-\mathrm{x}_{2}, \mathrm{y}_{1}-\mathrm{y}_{2}\right)\right\| \preceq\left\|\left(\eta \mathrm{x}_{1}, \eta \mathrm{x}_{2}\right)\right\| .
$$

Proof It is easy to check that $\left(\mathcal{X}^{\prime}, \nabla, \star\right)$ is a CVFMS with the CVFM defined by

$$
\left.\left.\nabla\left(\left(\mathrm{x}_{1}, \mathrm{y}_{1}\right)\right),\left(\mathrm{x}_{2}, \mathrm{y}_{2}\right)\right), \omega\right)=\frac{\omega}{\left.\left.\omega+\|\left(\mathrm{x}_{1}, \mathrm{y}_{1}\right)\right)-\left(\mathrm{x}_{2}, \mathrm{y}_{2}\right)\right) \|_{0}} \nu .
$$

If $\mathrm{t} \in \mathrm{J}$, from (1.1) and conditions $\left(\mathbb{H}_{1}\right)-\left(\mathbb{H}_{2}\right)$, for all $\left(\mathrm{x}_{1}, \mathrm{y}_{1}\right),\left(\mathrm{x}_{2}, \mathrm{y}_{2}\right) \in \mathrm{Y}^{\prime}$, we have

$$
\begin{aligned}
& \left|\mathcal{T}_{1}\left(\mathrm{x}_{1}, \mathrm{y}_{1}\right)(\mathrm{t})-\mathcal{T}_{2}\left(\mathrm{x}_{2}, \mathrm{y}_{2}\right)(\mathrm{t})\right| \\
& \quad \leq \frac{1}{\Gamma(\mu)} \int_{0}^{\mathrm{t}}(\mathrm{t}-\mathrm{s})^{\mu-1}\left|\mathrm{~h}\left(\mathrm{~s},{ }^{\mathrm{c}} \mathrm{D}^{\varrho} \mathrm{x}_{1}(\mathrm{~s}),{ }^{\mathrm{c}} \mathrm{D}^{\rho} \mathrm{y}_{1}(\mathrm{~s})\right)-\mathrm{h}\left(\mathrm{s},{ }^{\mathrm{c}} \mathrm{D}^{\varrho} \mathrm{x}_{2}(\mathrm{~s}),{ }^{\mathrm{c}} \mathrm{D}^{\rho} \mathrm{y}_{2}(\mathrm{~s})\right)\right| \mathrm{ds}
\end{aligned}
$$




$$
\begin{aligned}
& +\frac{\mathcal{Z}_{\mu} \mathrm{t}^{2}}{\Gamma(\mu-\omega)} \int_{\mathrm{t}_{\mathrm{j}}}^{\tau}(\tau-\mathrm{s})^{\mu-\omega-1}\left|\mathrm{~h}\left(\mathrm{~s},{ }^{\mathrm{c}} \mathrm{D}^{\varrho} \mathrm{x}_{1}(\mathrm{~s}),{ }^{\mathrm{c}} \mathrm{D}^{\rho} \mathrm{y}_{1}(\mathrm{~s})\right)-\mathrm{h}\left(\mathrm{s},{ }^{\mathrm{c}} \mathrm{D}^{\varrho} \mathrm{x}_{2}(\mathrm{~s}),{ }^{\mathrm{c}} \mathrm{D}^{\rho} \mathrm{y}_{2}(\mathrm{~s})\right)\right| \mathrm{ds} \\
& +\frac{\mathcal{Z}_{\mu} \mathrm{t}^{2} \tau^{1-\omega}}{\Gamma(\mu-1) \Gamma(2-\omega)} \\
& \times \sum_{\mathrm{j}=1}^{\mathrm{m}} \int_{\mathrm{t}_{\mathrm{j}-1}}^{\mathrm{t}_{\mathrm{j}}}\left(\mathrm{t}_{\mathrm{j}}-\mathrm{s}\right)^{\mu-2}\left|\mathrm{~h}\left(\mathrm{~s},{ }^{\mathrm{c}} \mathrm{D}^{\varrho} \mathrm{x}_{1}(\mathrm{~s}),{ }^{\mathrm{c}} \mathrm{D}^{\rho} \mathrm{y}_{1}(\mathrm{~s})\right)-\mathrm{h}\left(\mathrm{s},{ }^{\mathrm{c}} \mathrm{D}^{\varrho} \mathrm{x}_{2}(\mathrm{~s}),{ }^{\mathrm{c}} \mathrm{D}^{\rho} \mathrm{y}_{2}(\mathrm{~s})\right)\right| \mathrm{ds} \\
& +\frac{\mathcal{Z}_{\mu} \mathrm{t}^{2} \tau^{2-\omega}}{\Gamma(\mu-2) \Gamma(3-\omega)} \\
& \times \sum_{\mathrm{j}=1}^{\mathrm{m}} \int_{\mathrm{t}_{\mathrm{j}-1}}^{\mathrm{t}_{\mathrm{j}}}\left(\mathrm{t}_{\mathrm{j}}-\mathrm{s}\right)^{\mu-3}\left|\mathrm{~h}\left(\mathrm{~s},{ }^{\mathrm{c}} \mathrm{D}^{\varrho} \mathrm{x}_{1}(\mathrm{~s}),{ }^{\mathrm{c}} \mathrm{D}^{\rho} \mathrm{y}_{1}(\mathrm{~s})\right)-\mathrm{h}\left(\mathrm{s},{ }^{\mathrm{c}} \mathrm{D}^{\varrho} \mathrm{x}_{2}(\mathrm{~s}),{ }^{\mathrm{c}} \mathrm{D}^{\rho} \mathrm{y}_{2}(\mathrm{~s})\right)\right| \mathrm{ds} \\
& +\frac{\mathcal{Z}_{\mu} \mathrm{t}^{2} \tau^{1-\omega}}{\Gamma(\mu-2) \Gamma(2-\omega)} \\
& \times \sum_{\mathrm{j}=1}^{\mathrm{m}} \mathrm{t}_{\mathrm{j}} \int_{\mathrm{t}_{\mathrm{j}-1}}^{\mathrm{t}_{\mathrm{j}}}\left(\mathrm{t}_{\mathrm{j}}-\mathrm{s}\right)^{\mu-3}\left|\mathrm{~h}\left(\mathrm{~s},{ }^{\mathrm{c}} \mathrm{D}^{\varrho} \mathrm{x}_{1}(\mathrm{~s}),{ }^{\mathrm{c}} \mathrm{D}^{\rho} \mathrm{y}_{1}(\mathrm{~s})\right)-\mathrm{h}\left(\mathrm{s},{ }^{\mathrm{c}} \mathrm{D}^{\varrho} \mathrm{x}_{2}(\mathrm{~s}),{ }^{\mathrm{c}} \mathrm{D}^{\rho} \mathrm{y}_{2}(\mathrm{~s})\right)\right| \mathrm{ds} \\
& +\frac{\mathcal{Z}_{\mu} \mathrm{t}^{2} \tau^{1-\omega}}{\Gamma(2-\omega)} \sum_{\mathrm{j}=1}^{\mathrm{m}}\left|\mathcal{N}_{1 \mathrm{j}}\left(\mathrm{x}_{1}\left(\mathrm{t}_{\mathrm{j}}\right)\right)-\mathcal{N}_{1 \mathrm{j}}\left(\mathrm{x}_{2}\left(\mathrm{t}_{\mathrm{j}}\right)\right)\right| \\
& +\frac{\mathcal{Z}_{\mu} \mathrm{t}^{2} \tau^{2-\omega}}{\Gamma(3-\omega)} \sum_{\mathrm{j}=1}^{\mathrm{m}}\left|\mathcal{O}_{1 \mathrm{j}}\left(\mathrm{x}_{1}\left(\mathrm{t}_{\mathrm{j}}\right)\right)-\mathcal{O}_{1 \mathrm{j}}\left(\mathrm{x}_{2}\left(\mathrm{t}_{\mathrm{j}}\right)\right)\right| \\
& +\frac{\mathcal{Z}_{\mu} \mathrm{t}^{2}}{\Gamma(\mu-2)} \int_{\mathrm{t}_{\mathrm{m}}}^{1}(1-\mathrm{s})^{\mu-3}\left|\mathrm{~h}\left(\mathrm{~s},{ }^{\mathrm{c}} \mathrm{D}^{\varrho} \mathrm{x}_{1}(\mathrm{~s}),{ }^{\mathrm{c}} \mathrm{D}^{\rho} \mathrm{y}_{1}(\mathrm{~s})\right)-\mathrm{h}\left(\mathrm{s},{ }^{\mathrm{c}} \mathrm{D}^{\varrho} \mathrm{x}_{2}(\mathrm{~s}),{ }^{\mathrm{c}} \mathrm{D}^{\rho} \mathrm{y}_{2}(\mathrm{~s})\right)\right| \mathrm{ds} \\
& +\frac{\mathcal{Z}_{\mu} \mathrm{t}^{2}}{\Gamma(\mu-2)} \\
& \times \sum_{\mathrm{j}=1}^{\mathrm{m}} \mathrm{t}_{\mathrm{j}} \int_{\mathrm{t}_{\mathrm{j}-1}}^{\mathrm{t}_{\mathrm{j}}}\left(\mathrm{t}_{\mathrm{j}}-\mathrm{s}\right)^{\mu-3}\left|\mathrm{~h}\left(\mathrm{~s},{ }^{\mathrm{c}} \mathrm{D}^{\varrho} \mathrm{x}_{1}(\mathrm{~s}),{ }^{\mathrm{c}} \mathrm{D}^{\rho} \mathrm{y}_{1}(\mathrm{~s})\right)-\mathrm{h}\left(\mathrm{s},{ }^{\mathrm{c}} \mathrm{D}^{\varrho} \mathrm{x}_{2}(\mathrm{~s}),{ }^{\mathrm{c}} \mathrm{D}^{\rho} \mathrm{y}_{2}(\mathrm{~s})\right)\right| \mathrm{ds} \\
& +\frac{\mathcal{Z}_{\mu} \mathrm{t}^{2} \tau^{1-\omega}}{\Gamma(2-\omega)} \sum_{\mathrm{j}=1}^{\mathrm{m}}\left|\mathrm{t}_{\mathrm{j}}\right|\left|\mathcal{O}_{1 \mathrm{j}}\left(\mathrm{x}_{1}\left(\mathrm{t}_{\mathrm{j}}\right)\right)-\mathcal{O}_{1 \mathrm{j}}\left(\mathrm{x}_{2}\left(\mathrm{t}_{\mathrm{j}}\right)\right)\right| \\
& +\mathcal{Z}_{\mu} \mathrm{t}^{2} \sum_{\mathrm{j}=1}^{\mathrm{m}}\left|\mathcal{O}_{1 \mathrm{j}}\left(\mathrm{x}_{1}\left(\mathrm{t}_{\mathrm{j}}\right)\right)-\mathcal{O}_{1 \mathrm{j}}\left(\mathrm{x}_{2}\left(\mathrm{t}_{\mathrm{j}}\right)\right)\right| \\
& \leq \frac{1}{\Gamma(\mu+1)}\left[\left.\mathrm{A}_{1}\right|^{\mathrm{c}} \mathrm{D}^{\varrho} \mathrm{x}_{1}(\mathrm{~s})-{ }^{\mathrm{c}} \mathrm{D}^{\varrho} \mathrm{x}_{2}(\mathrm{~s})\left|+\mathrm{A}_{2}\right|^{\mathrm{c}} \mathrm{D}^{\rho} \mathrm{y}_{1}(\mathrm{~s})-{ }^{\mathrm{c}} \mathrm{D}^{\rho} \mathrm{y}_{2}(\mathrm{~s}) \mid\right] \\
& +\frac{\mathcal{Z}_{\mu}}{\Gamma(\mu-\omega+1)}\left[\mathrm{A}_{1}\left|{ }^{\mathrm{c}} \mathrm{D}^{\varrho} \mathrm{x}_{1}(\mathrm{~s})-{ }^{\mathrm{c}} \mathrm{D}^{\varrho} \mathrm{x}_{2}(\mathrm{~s})\right|+\mathrm{A}_{2}\left|{ }^{\mathrm{c}} \mathrm{D}^{\rho} \mathrm{y}_{1}(\mathrm{~s})-{ }^{\mathrm{c}} \mathrm{D}^{\rho} \mathrm{y}_{2}(\mathrm{~s})\right|\right] \\
& +\frac{\mathcal{Z}_{\mu} \tau^{1-\omega}}{\Gamma(\mu) \Gamma(2-\omega)}\left[\mathrm{A}_{1}\left|{ }^{\mathrm{c}} \mathrm{D}^{\varrho} \mathrm{x}_{1}(\mathrm{~s})-{ }^{\mathrm{c}} \mathrm{D}^{\varrho} \mathrm{x}_{2}(\mathrm{~s})\right|+\left.\mathrm{A}_{2}\right|^{\mathrm{c}} \mathrm{D}^{\rho} \mathrm{y}_{1}(\mathrm{~s})-{ }^{\mathrm{c}} \mathrm{D}^{\rho} \mathrm{y}_{2}(\mathrm{~s}) \mid\right] \\
& +\frac{\mathcal{Z}_{\mu} \tau^{2-\omega}}{\Gamma(\mu-1) \Gamma(3-\omega)}\left[\mathrm{A}_{1}\left|{ }^{\mathrm{c}} \mathrm{D}^{\varrho} \mathrm{x}_{1}(\mathrm{~s})-{ }^{\mathrm{c}} \mathrm{D}^{\varrho} \mathrm{x}_{2}(\mathrm{~s})\right|+\mathrm{A}_{2}\left|{ }^{\mathrm{c}} \mathrm{D}^{\rho} \mathrm{y}_{1}(\mathrm{~s})-{ }^{\mathrm{c}} \mathrm{D}^{\rho} \mathrm{y}_{2}(\mathrm{~s})\right|\right] \\
& +\frac{\mathcal{Z}_{\mu} \tau^{1-\omega}}{\Gamma(\mu-1) \Gamma(2-\omega)}\left[\left.\mathrm{A}_{1}\right|^{\mathrm{c}} \mathrm{D}^{\varrho} \mathrm{x}_{1}(\mathrm{~s})-{ }^{\mathrm{c}} \mathrm{D}^{\varrho} \mathrm{x}_{2}(\mathrm{~s})\left|+\mathrm{A}_{2}\right|^{\mathrm{c}} \mathrm{D}^{\rho} \mathrm{y}_{1}(\mathrm{~s})-{ }^{\mathrm{c}} \mathrm{D}^{\rho} \mathrm{y}_{2}(\mathrm{~s}) \mid\right]
\end{aligned}
$$




$$
\begin{aligned}
& +\frac{\mathcal{Z}_{\mu} \tau^{1-\omega}}{\Gamma(2-\omega)} \sum_{\mathrm{j}=1}^{\mathrm{m}}\left|\mathcal{N}_{1 \mathrm{j}}\left(\mathrm{x}_{1}\left(\mathrm{t}_{\mathrm{j}}\right)\right)-\mathcal{N}_{1 \mathrm{j}}\left(\mathrm{x}_{2}\left(\mathrm{t}_{\mathrm{j}}\right)\right)\right| \\
& +\frac{\mathcal{Z}_{\mu} \tau^{2-\omega}}{\Gamma(3-\omega)} \sum_{j=1}^{m}\left|\mathcal{O}_{1 j}\left(x_{1}\left(t_{j}\right)\right)-\mathcal{O}_{1 j}\left(x_{2}\left(t_{j}\right)\right)\right| \\
& +\frac{\mathcal{Z}_{\mu}}{\Gamma(\mu-1)}\left[\mathrm{A}_{1}\left|{ }^{\mathrm{c}} \mathrm{D}^{\varrho} \mathrm{x}_{1}(\mathrm{~s})-{ }^{\mathrm{c}} \mathrm{D}^{\varrho} \mathrm{x}_{2}(\mathrm{~s})\right|+\mathrm{A}_{2}\left|{ }^{\mathrm{c}} \mathrm{D}^{\rho} \mathrm{y}_{1}(\mathrm{~s})-{ }^{\mathrm{c}} \mathrm{D}^{\rho} \mathrm{y}_{2}(\mathrm{~s})\right|\right] \\
& +\frac{\mathcal{Z}_{\mu}}{\Gamma(\mu-1)}\left[\mathrm{A}_{1}\left|{ }^{\mathrm{c}} \mathrm{D}^{\varrho} \mathrm{x}_{1}(\mathrm{~s})-{ }^{\mathrm{c}} \mathrm{D}^{\varrho} \mathrm{x}_{2}(\mathrm{~s})\right|+\mathrm{A}_{2}\left|{ }^{\mathrm{c}} \mathrm{D}^{\rho} \mathrm{y}_{1}(\mathrm{~s})-{ }^{\mathrm{c}} \mathrm{D}^{\rho} \mathrm{y}_{2}(\mathrm{~s})\right|\right] \\
& +\frac{\mathcal{Z}_{\mu} \mathrm{t}^{2} \tau^{1-\omega}}{\Gamma(2-\omega)} \sum_{\mathrm{j}=1}^{\mathrm{m}}\left|\mathrm{t}_{\mathrm{j}}\right|\left|\mathcal{O}_{1 \mathrm{j}}\left(\mathrm{x}_{1}\left(\mathrm{t}_{\mathrm{j}}\right)\right)-\mathcal{O}_{1 j}\left(\mathrm{x}_{2}\left(\mathrm{t}_{\mathrm{j}}\right)\right)\right|+\mathcal{Z}_{\mu} \sum_{\mathrm{j}=1}^{\mathrm{m}}\left|\mathcal{O}_{1 \mathrm{j}}\left(\mathrm{x}_{1}\left(\mathrm{t}_{\mathrm{j}}\right)\right)-\mathcal{O}_{1 \mathrm{j}}\left(\mathrm{x}_{2}\left(\mathrm{t}_{\mathrm{j}}\right)\right)\right| \\
& \leq \frac{1}{\Gamma(\mu+1)}\left[\mathrm{A}_{1}\left\|\mathrm{x}_{1}-\mathrm{x}_{2}\right\|_{0}+\mathrm{A}_{2}\left\|\mathrm{y}_{1}-\mathrm{y}_{2}\right\|_{0}\right] \\
& +\frac{\mathcal{Z}_{\mu}}{\Gamma(\mu-\omega+1)}\left[\mathrm{A}_{1}\left\|\mathrm{x}_{1}-\mathrm{x}_{2}\right\|_{0}+\mathrm{A}_{2}\left\|\mathrm{y}_{1}-\mathrm{y}_{2}\right\|_{0}\right] \\
& +\frac{\mathcal{Z}_{\mu} \tau^{1-\omega}}{\Gamma(\mu) \Gamma(2-\omega)}\left[\mathrm{A}_{1}\left\|\mathrm{x}_{1}-\mathrm{x}_{2}\right\|_{0}+\mathrm{A}_{2}\left\|\mathrm{y}_{1}-\mathrm{y}_{2}\right\|_{0}\right] \\
& +\frac{\mathcal{Z}_{\mu} \tau^{2-\omega}}{\Gamma(\mu-1) \Gamma(3-\omega)}\left[\mathrm{A}_{1}\left\|\mathrm{x}_{1}-\mathrm{x}_{2}\right\|_{0}+\mathrm{A}_{2}\left\|\mathrm{y}_{1}-\mathrm{y}_{2}\right\|_{0}\right] \\
& +\frac{\mathcal{Z}_{\mu} \tau^{1-\omega}}{\Gamma(\mu-1) \Gamma(2-\omega)}\left[\mathrm{A}_{1}\left\|\mathrm{x}_{1}-\mathrm{x}_{2}\right\|_{0}+\mathrm{A}_{2}\left\|\mathrm{y}_{1}-\mathrm{y}_{2}\right\|_{0}\right]+\frac{\mathcal{Z}_{\mu} \tau^{1-\omega}}{\Gamma(2-\omega)} \sum_{\mathrm{j}=1}^{\mathrm{m}} \check{\mathrm{L}}_{1 \mathrm{j}}\left\|\mathrm{x}_{1}-\mathrm{x}_{2}\right\|_{0} \\
& +\frac{\mathcal{Z}_{\mu} \tau^{2-\omega}}{\Gamma(3-\omega)} \sum_{\mathrm{j}=1}^{\mathrm{m}} \check{\mathrm{L}}_{1 \mathrm{j}}\left\|\mathrm{x}_{1}-\mathrm{x}_{2}\right\|_{0}+\frac{\mathcal{Z}_{\mu}}{\Gamma(\mu-1)}\left[\mathrm{A}_{1}\left\|\mathrm{x}_{1}-\mathrm{x}_{2}\right\|_{0}+\mathrm{A}_{2}\left\|\mathrm{y}_{1}-\mathrm{y}_{2}\right\|_{0}\right] \\
& +\frac{\mathcal{Z}_{\mu}}{\Gamma(\mu-1)}\left[\mathrm{A}_{1}\left\|\mathrm{x}_{1}-\mathrm{x}_{2}\right\|_{0}+\mathrm{A}_{2}\left\|\mathrm{y}_{1}-\mathrm{y}_{2}\right\|_{0}\right]+\frac{\mathcal{Z}_{\mu} \tau^{1-\omega}}{\Gamma(2-\omega)} \sum_{\mathrm{j}=1}^{\mathrm{m}}\left|\mathrm{t}_{\mathrm{j}}\right| \check{\mathrm{L}}_{1 \mathrm{j}}\left\|\mathrm{x}_{1}-\mathrm{x}_{2}\right\|_{0} \\
& +\mathcal{Z}_{\mu} \sum_{j=1}^{\mathrm{m}} \check{\mathrm{L}}_{1 \mathrm{j}}\left\|\mathrm{x}_{1}-\mathrm{x}_{2}\right\|_{0} \\
& \leq \frac{1}{\Gamma(\mu+1)}\left[\mathrm{A}_{1}+\mathrm{A}_{2}\right]\left\|\left(\mathrm{x}_{1}-\mathrm{x}_{2}, \mathrm{y}_{1}-\mathrm{y}_{2}\right)\right\| \\
& +\frac{\mathcal{Z}_{\mu}}{\Gamma(\mu-\omega+1)}\left[\mathrm{A}_{1}+\mathrm{A}_{2}\right]\left\|\left(\mathrm{x}_{1}-\mathrm{x}_{2}, \mathrm{y}_{1}-\mathrm{y}_{2}\right)\right\| \\
& +\frac{\mathcal{Z}_{\mu} \tau^{1-\omega}}{\Gamma(\mu) \Gamma(2-\omega)}\left[\mathrm{A}_{1}+\mathrm{A}_{2}\right]\left\|\left(\mathrm{x}_{1}-\mathrm{x}_{2}, \mathrm{y}_{1}-\mathrm{y}_{2}\right)\right\| \\
& +\frac{\mathcal{Z}_{\mu} \tau^{2-\omega}}{\Gamma(\mu-1) \Gamma(3-\omega)}\left[\mathrm{A}_{1}+\mathrm{A}_{2}\right]\left\|\left(\mathrm{x}_{1}-\mathrm{x}_{2}, \mathrm{y}_{1}-\mathrm{y}_{2}\right)\right\| \\
& +\frac{\mathcal{Z}_{\mu} \tau^{1-\omega}}{\Gamma(\mu-1) \Gamma(2-\omega)}\left[\mathrm{A}_{1}+\mathrm{A}_{2}\right]\left\|\left(\mathrm{x}_{1}-\mathrm{x}_{2}, \mathrm{y}_{1}-\mathrm{y}_{2}\right)\right\|+\frac{\mathcal{Z}_{\mu} \tau{ }^{1-\omega}}{\Gamma(2-\omega)} \sum_{\mathrm{j}=1}^{\mathrm{m}} \check{\mathrm{L}}_{1 \mathrm{j}}\left\|\mathrm{x}_{1}-\mathrm{x}_{2}\right\|_{0} \\
& +\frac{\mathcal{Z}_{\mu} \tau^{2-\omega}}{\Gamma(3-\omega)} \sum_{\mathrm{j}=1}^{\mathrm{m}} \check{\mathrm{L}}_{1 \mathrm{j}}\left\|\mathrm{x}_{1}-\mathrm{x}_{2}\right\|_{0}+\frac{\mathcal{Z}_{\mu}}{\Gamma(\mu-1)}\left[\mathrm{A}_{1}+\mathrm{A}_{2}\right]\left\|\left(\mathrm{x}_{1}-\mathrm{x}_{2}, \mathrm{y}_{1}-\mathrm{y}_{2}\right)\right\|
\end{aligned}
$$




$$
\begin{aligned}
& +\frac{\mathcal{Z}_{\mu}}{\Gamma(\mu-1)}\left[\mathrm{A}_{1}+\mathrm{A}_{2}\right]\left\|\left(\mathrm{x}_{1}-\mathrm{x}_{2}, \mathrm{y}_{1}-\mathrm{y}_{2}\right)\right\|+\frac{\mathcal{Z}_{\mu} \tau^{1-\omega}}{\Gamma(2-\omega)} \sum_{\mathrm{j}=1}^{\mathrm{m}}\left|\mathrm{t}_{\mathrm{j}}\right| \check{\mathrm{L}}_{1 \mathrm{j}}\left\|\left(\mathrm{x}_{1}-\mathrm{x}_{2}, \mathrm{y}_{1}-\mathrm{y}_{2}\right)\right\| \\
& +\mathcal{Z}_{\mu} \mathrm{t}^{2} \sum_{\mathrm{j}=1}^{\mathrm{m}} \check{\mathrm{L}}_{1 \mathrm{j}}\left\|\left(\mathrm{x}_{1}-\mathrm{x}_{2}, \mathrm{y}_{1}-\mathrm{y}_{2}\right)\right\| \\
& \leq \mathcal{Z}_{\mu}\left[( \mathrm { A } _ { 1 } + \mathrm { A } _ { 2 } ) \left(\frac{1}{\mathcal{Z}_{\mu} \Gamma(\mu+1)}+\frac{1}{\Gamma(\mu-\omega+1)}+\frac{\tau^{1-\omega}}{\Gamma(\mu) \Gamma(2-\omega)}+\frac{\tau^{2-\omega}}{\Gamma(\mu-1) \Gamma(3-\omega)}\right.\right. \\
& \left.+\frac{\tau^{1-\omega}}{\Gamma(\mu-1) \Gamma(2-\omega)}+\frac{2}{\Gamma(\mu-1)}\right)+\frac{\tau^{1-\omega}}{\Gamma(2-\omega)} \sum_{\mathrm{j}=1}^{\mathrm{m}} \check{\mathrm{L}}_{1 \mathrm{j}}+\frac{\tau^{2-\omega}}{\Gamma(3-\omega)} \sum_{\mathrm{j}=1}^{\mathrm{m}} \check{\mathrm{L}}_{1 \mathrm{j}} \\
& \left.+\frac{\tau^{1-\omega}}{\Gamma(2-\omega)} \sum_{\mathrm{j}=1}^{\mathrm{m}} \check{\mathrm{L}}_{1 \mathrm{j}}+\sum_{\mathrm{j}=1}^{\mathrm{m}} \check{\mathrm{L}}_{1 \mathrm{j}}\right]\left\|\left(\mathrm{x}_{1}-\mathrm{x}_{2}, \mathrm{y}_{1}-\mathrm{y}_{2}\right)\right\|, \quad \mathrm{t} \in\left[0, \mathrm{t}_{1}\right] .
\end{aligned}
$$

When $t \in\left[t_{m}, t_{m+1}\right]$, then

$$
\begin{aligned}
& \left|\mathcal{T}_{1}\left(\mathrm{x}_{1}, \mathrm{y}_{1}\right)(\mathrm{t})-\mathcal{T}_{2}\left(\mathrm{x}_{2}, \mathrm{y}_{2}\right)(\mathrm{t})\right| \\
& \leq \frac{1}{\Gamma(\mu)} \int_{\mathrm{t}_{\mathrm{m}}}^{\mathrm{t}}(\mathrm{t}-\mathrm{s})^{\mu-1}\left|\mathrm{~h}\left(\mathrm{~s},{ }^{\mathrm{c}} \mathrm{D}^{\varrho} \mathrm{x}_{1}(\mathrm{~s}),{ }^{\mathrm{c}} \mathrm{D}^{\rho} \mathrm{y}_{1}(\mathrm{~s})\right)-\mathrm{h}\left(\mathrm{s},{ }^{\mathrm{c}} \mathrm{D}^{\varrho} \mathrm{x}_{2}(\mathrm{~s}),{ }^{\mathrm{c}} \mathrm{D}^{\rho} \mathrm{y}_{2}(\mathrm{~s})\right)\right| \mathrm{ds} \\
& +\frac{1}{\Gamma(\mu)} \sum_{\mathrm{j}=1}^{\mathrm{m}} \int_{\mathrm{t}_{\mathrm{j}-1}}^{\mathrm{t}_{\mathrm{j}}}\left(\mathrm{t}_{\mathrm{j}}-\mathrm{s}\right)^{\mu-1}\left|\mathrm{~h}\left(\mathrm{~s},{ }^{\mathrm{c}} \mathrm{D}^{\varrho} \mathrm{x}_{1}(\mathrm{~s}),{ }^{\mathrm{c}} \mathrm{D}^{\rho} \mathrm{y}_{1}(\mathrm{~s})\right)-\mathrm{h}\left(\mathrm{s},{ }^{\mathrm{c}} \mathrm{D}^{\varrho} \mathrm{x}_{2}(\mathrm{~s}),{ }^{\mathrm{c}} \mathrm{D}^{\rho} \mathrm{y}_{2}(\mathrm{~s})\right)\right| \mathrm{ds} \\
& +\frac{1}{\Gamma(\mu-1)} \sum_{\mathrm{j}=1}^{\mathrm{m}}\left|\left(\mathrm{t}-\mathrm{t}_{\mathrm{j}}\right)\right| \\
& \times \int_{\mathrm{t}_{\mathrm{j}-1}}^{\mathrm{t}_{\mathrm{j}}}\left(\mathrm{t}_{\mathrm{j}}-\mathrm{s}\right)^{\mu-2}\left|\mathrm{~h}\left(\mathrm{~s},{ }^{\mathrm{c}} \mathrm{D}^{\varrho} \mathrm{x}_{1}(\mathrm{~s}),{ }^{\mathrm{c}} \mathrm{D}^{\rho} \mathrm{y}_{1}(\mathrm{~s})\right)-\mathrm{h}\left(\mathrm{s},{ }^{\mathrm{c}} \mathrm{D}^{\varrho} \mathrm{x}_{2}(\mathrm{~s}),{ }^{\mathrm{c}} \mathrm{D}^{\rho} \mathrm{y}_{2}(\mathrm{~s})\right)\right| \mathrm{ds} \\
& +\frac{1}{2 \Gamma(\mu-2)} \sum_{\mathrm{j}=1}^{\mathrm{m}}\left|\left(\mathrm{t}-\mathrm{t}_{\mathrm{j}}\right)^{2}\right| \\
& \times \int_{\mathrm{t}_{\mathrm{j}-1}}^{\mathrm{t}_{\mathrm{j}}}\left(\mathrm{t}_{\mathrm{j}}-\mathrm{s}\right)^{\mu-3}\left|\mathrm{~h}\left(\mathrm{~s},{ }^{\mathrm{c}} \mathrm{D}^{\varrho} \mathrm{x}_{1}(\mathrm{~s}),{ }^{\mathrm{c}} \mathrm{D}^{\rho} \mathrm{y}_{1}(\mathrm{~s})\right)-\mathrm{h}\left(\mathrm{s},{ }^{\mathrm{c}} \mathrm{D}^{\varrho} \mathrm{x}_{2}(\mathrm{~s}),{ }^{\mathrm{c}} \mathrm{D}^{\rho} \mathrm{y}_{2}(\mathrm{~s})\right)\right| \mathrm{ds} \\
& +\sum_{j=1}^{m}\left|\mathcal{M}_{1 j}\left(x_{1}\left(t_{j}\right)\right)-\mathcal{M}_{1 j}\left(x_{2}\left(t_{j}\right)\right)\right|+\sum_{j=1}^{m}\left|\left(t-t_{j}\right)\right|\left|\mathcal{N}_{1 j}\left(x_{1}\left(t_{j}\right)\right)-\mathcal{N}_{1 j}\left(x_{2}\left(t_{j}\right)\right)\right| \\
& +\frac{\mathcal{Z}_{\mu} \mathrm{t}^{2}}{\Gamma(\mu-\omega)} \int_{\mathrm{t}_{\mathrm{j}}}^{\tau}(\tau-\mathrm{s})^{\mu-\omega-1}\left|\mathrm{~h}\left(\mathrm{~s},{ }^{\mathrm{c}} \mathrm{D}^{\varrho} \mathrm{x}_{1}(\mathrm{~s}),{ }^{\mathrm{c}} \mathrm{D}^{\rho} \mathrm{y}_{1}(\mathrm{~s})\right)-\mathrm{h}\left(\mathrm{s},{ }^{\mathrm{c}} \mathrm{D}^{\varrho} \mathrm{x}_{2}(\mathrm{~s}),{ }^{\mathrm{c}} \mathrm{D}^{\rho} \mathrm{y}_{2}(\mathrm{~s})\right)\right| \mathrm{ds} \\
& +\frac{\mathcal{Z}_{\mu} \mathrm{t}^{2} \tau^{1-\omega}}{\Gamma(\mu-1) \Gamma(2-\omega)} \\
& \times \sum_{\mathrm{j}=1}^{\mathrm{m}} \int_{\mathrm{t}_{\mathrm{j}-1}}^{\mathrm{t}_{\mathrm{j}}}\left(\mathrm{t}_{\mathrm{j}}-\mathrm{s}\right)^{\mu-2}\left|\mathrm{~h}\left(\mathrm{~s},{ }^{\mathrm{c}} \mathrm{D}^{\varrho} \mathrm{x}_{1}(\mathrm{~s}),{ }^{\mathrm{c}} \mathrm{D}^{\rho} \mathrm{y}_{1}(\mathrm{~s})\right)-\mathrm{h}\left(\mathrm{s},{ }^{\mathrm{c}} \mathrm{D}^{\varrho} \mathrm{x}_{2}(\mathrm{~s}),{ }^{\mathrm{c}} \mathrm{D}^{\rho} \mathrm{y}_{2}(\mathrm{~s})\right)\right| \mathrm{ds} \\
& +\frac{\mathcal{Z}_{\mu} \mathrm{t}^{2} \tau^{2-\omega}}{\Gamma(\mu-2) \Gamma(3-\omega)} \\
& \times \sum_{\mathrm{j}=1}^{\mathrm{m}} \int_{\mathrm{t}_{\mathrm{j}-1}}^{\mathrm{t}_{\mathrm{j}}}\left(\mathrm{t}_{\mathrm{j}}-\mathrm{s}\right)^{\mu-3}\left|\mathrm{~h}\left(\mathrm{~s},{ }^{\mathrm{c}} \mathrm{D}^{\varrho} \mathrm{x}_{1}(\mathrm{~s}),{ }^{\mathrm{c}} \mathrm{D}^{\rho} \mathrm{y}_{1}(\mathrm{~s})\right)-\mathrm{h}\left(\mathrm{s},{ }^{\mathrm{c}} \mathrm{D}^{\varrho} \mathrm{x}_{2}(\mathrm{~s}),{ }^{\mathrm{c}} \mathrm{D}^{\rho} \mathrm{y}_{2}(\mathrm{~s})\right)\right| \mathrm{ds}
\end{aligned}
$$




$$
\begin{aligned}
& +\frac{\mathcal{Z}_{\mu} \mathrm{t}^{2} \tau^{1-\omega}}{\Gamma(\mu-2) \Gamma(2-\omega)} \\
& \times \sum_{\mathrm{j}=1}^{\mathrm{m}} \mathrm{t}_{\mathrm{j}} \int_{\mathrm{t}_{\mathrm{j}-1}}^{\mathrm{t}_{j}}\left(\mathrm{t}_{\mathrm{j}}-\mathrm{s}\right)^{\mu-3}\left|\mathrm{~h}\left(\mathrm{~s},{ }^{\mathrm{c}} \mathrm{D}^{\varrho} \mathrm{x}_{1}(\mathrm{~s}),{ }^{\mathrm{c}} \mathrm{D}^{\rho} \mathrm{y}_{1}(\mathrm{~s})\right)-\mathrm{h}\left(\mathrm{s},{ }^{\mathrm{c}} \mathrm{D}^{\varrho} \mathrm{x}_{2}(\mathrm{~s}),{ }^{\mathrm{c}} \mathrm{D}^{\rho} \mathrm{y}_{2}(\mathrm{~s})\right)\right| \mathrm{ds} \\
& +\frac{\mathcal{Z}_{\mu} \mathrm{t}^{2} \tau^{1-\omega}}{\Gamma(2-\omega)} \sum_{\mathrm{j}=1}^{\mathrm{m}}\left|\mathcal{N}_{1 \mathrm{j}}\left(\mathrm{x}_{1}\left(\mathrm{t}_{\mathrm{j}}\right)\right)-\mathcal{N}_{1 \mathrm{j}}\left(\mathrm{x}_{2}\left(\mathrm{t}_{\mathrm{j}}\right)\right)\right| \\
& +\frac{\mathcal{Z}_{\mu} t^{2} \tau^{2-\omega}}{\Gamma(3-\omega)} \sum_{j=1}^{m}\left|\mathcal{O}_{1 j}\left(x_{1}\left(t_{j}\right)\right)-\mathcal{O}_{1 j}\left(x_{2}\left(t_{j}\right)\right)\right| \\
& +\frac{\mathcal{Z}_{\mu} \mathrm{t}^{2}}{\Gamma(\mu-2)} \int_{\mathrm{t}_{\mathrm{m}}}^{1}(1-\mathrm{s})^{\mu-3}\left|\mathrm{~h}\left(\mathrm{~s},{ }^{\mathrm{c}} \mathrm{D}^{\varrho} \mathrm{x}_{1}(\mathrm{~s}),{ }^{\mathrm{c}} \mathrm{D}^{\rho} \mathrm{y}_{1}(\mathrm{~s})\right)-\mathrm{h}\left(\mathrm{s}^{\mathrm{c}}{ }^{\mathrm{c}} \mathrm{D}^{\varrho} \mathrm{x}_{2}(\mathrm{~s}),{ }^{\mathrm{c}} \mathrm{D}^{\rho} \mathrm{y}_{2}(\mathrm{~s})\right)\right| \mathrm{ds} \\
& +\frac{\mathcal{Z}_{\mu} \mathrm{t}^{2}}{\Gamma(\mu-2)} \\
& \times \sum_{\mathrm{j}=1}^{\mathrm{m}} \mathrm{t}_{\mathrm{j}} \int_{\mathrm{t}_{\mathrm{j}-1}}^{\mathrm{t}_{\mathrm{j}}}\left(\mathrm{t}_{\mathrm{j}}-\mathrm{s}\right)^{\mu-3}\left|\mathrm{~h}\left(\mathrm{~s},{ }^{\mathrm{c}} \mathrm{D}^{\varrho} \mathrm{x}_{1}(\mathrm{~s}),{ }^{\mathrm{c}} \mathrm{D}^{\rho} \mathrm{y}_{1}(\mathrm{~s})\right)-\mathrm{h}\left(\mathrm{s},{ }^{\mathrm{c}} \mathrm{D}^{\varrho} \mathrm{x}_{2}(\mathrm{~s}),{ }^{\mathrm{c}} \mathrm{D}^{\rho} \mathrm{y}_{2}(\mathrm{~s})\right)\right| \mathrm{ds} \\
& +\frac{\mathcal{Z}_{\mu} \mathrm{t}^{2} \tau^{1-\omega}}{\Gamma(2-\omega)} \sum_{\mathrm{j}=1}^{\mathrm{m}}\left|\mathrm{t}_{\mathrm{j}}\right|\left|\mathcal{O}_{1 \mathrm{j}}\left(\mathrm{x}_{1}\left(\mathrm{t}_{\mathrm{j}}\right)\right)-\mathcal{O}_{1 \mathrm{j}}\left(\mathrm{x}_{2}\left(\mathrm{t}_{\mathrm{j}}\right)\right)\right| \\
& +\mathcal{Z}_{\mu} \mathrm{t}^{2} \sum_{\mathrm{j}=1}^{\mathrm{m}}\left|\mathcal{O}_{1 \mathrm{j}}\left(\mathrm{x}_{1}\left(\mathrm{t}_{\mathrm{j}}\right)\right)-\mathcal{O}_{1 \mathrm{j}}\left(\mathrm{x}_{2}\left(\mathrm{t}_{\mathrm{j}}\right)\right)\right| \\
& +\sum_{j=1}^{m} \frac{\left|\left(t-t_{j}\right)^{2}\right|}{2}\left|\mathcal{O}_{1 j}\left(x_{1}\left(t_{j}\right)\right)-\mathcal{O}_{1 j}\left(x_{2}\left(t_{j}\right)\right)\right|
\end{aligned}
$$

By taking maximum and using $\left(\mathbb{H}_{3}\right),\left(\mathbb{H}_{4}\right)$ in $(4.4)$, we obtain

$$
\begin{aligned}
\leq & \frac{1}{\Gamma(\mu+1)}\left[\mathrm{A}_{1}\left\|\mathrm{x}_{1}-\mathrm{x}_{2}\right\|_{0}+\mathrm{A}_{2}\left\|_{\mathrm{y}_{1}}-\mathrm{y}_{2}\right\|_{0}\right]+\frac{1}{\Gamma(\mu+1)}\left[\mathrm{A}_{1}\left\|\mathrm{x}_{1}-\mathrm{x}_{2}\right\|_{0}+\mathrm{A}_{2}\left\|\mathrm{y}_{1}-\mathrm{y}_{2}\right\|_{0}\right] \\
& +\frac{1}{\Gamma(\mu)}\left[\mathrm{A}_{1}\left\|\mathrm{x}_{1}-\mathrm{x}_{2}\right\|_{0}+\mathrm{A}_{2}\left\|\mathrm{y}_{1}-\mathrm{y}_{2}\right\|_{0}\right]+\frac{1}{2 \Gamma(\mu-1)}\left[\mu_{1}\left\|\mathrm{x}_{1}-\mathrm{x}_{2}\right\|_{0}+\mu_{2}\left\|\mathrm{y}_{1}-\mathrm{y}_{2}\right\|_{0}\right] \\
& +\sum_{\mathrm{j}=1}^{\mathrm{m}} \mathrm{L}_{1 j}\left\|\mathrm{x}_{1}-\mathrm{x}_{2}\right\|_{0}+\sum_{\mathrm{j}=1}^{\mathrm{m}} \hat{\mathrm{L}}_{1 \mathrm{j}}\left\|\mathrm{x}_{1}-\mathrm{x}_{2}\right\|_{0} \\
& +\frac{\mathcal{Z}_{\mu}}{\Gamma(\mu-\omega+1)}\left[\mathrm{A}_{1}\left\|\mathrm{x}_{1}-\mathrm{x}_{2}\right\|_{0}+\mathrm{A}_{2}\left\|\mathrm{y}_{1}-\mathrm{y}_{2}\right\|_{0}\right] \\
& +\frac{\mathcal{Z}_{\mu} \tau^{1-\omega}}{\Gamma(\mu) \Gamma(2-\omega)}\left[\mathrm{A}_{1}\left\|\mathrm{x}_{1}-\mathrm{x}_{2}\right\|_{0}+\mathrm{A}_{2}\left\|\mathrm{y}_{1}-\mathrm{y}_{2}\right\|_{0}\right] \\
& +\frac{\mathcal{Z}_{\mu} \tau^{2-\omega}}{\Gamma(\mu-1) \Gamma(3-\omega)}\left[\mathrm{A}_{1}\left\|\mathrm{x}_{1}-\mathrm{x}_{2}\right\|_{0}+\mathrm{A}_{2}\left\|\mathrm{y}_{1}-\mathrm{y}_{2}\right\|_{0}\right] \\
& +\frac{\mathcal{Z}_{\mu} \tau^{1-\omega}}{\Gamma(\mu-1) \Gamma(2-\omega)}\left[\mathrm{A}_{1}\left\|\mathrm{x}_{1}-\mathrm{x}_{2}\right\|_{0}+\mathrm{A}_{2}\left\|\mathrm{y}_{1}-\mathrm{y}_{2}\right\|_{0}\right]+\frac{\mathcal{Z}_{\mu} \tau \tau^{1-\omega}}{\Gamma(2-\omega)} \sum_{\mathrm{j}=1}^{\mathrm{m}} \hat{\mathrm{L}}_{1 j}\left\|\mathrm{x}_{1}-\mathrm{x}_{2}\right\|_{0} \\
& +\frac{\mathcal{Z}_{\mu} \tau^{2-\omega}}{\Gamma(3-\omega)} \sum_{\mathrm{j}=1}^{\mathrm{m}} \check{\mathrm{L}}{ }_{1 j}\left\|\mathrm{x}_{1}-\mathrm{x}_{2}\right\|_{0}+\frac{\mathcal{Z}_{\mu}}{\Gamma(\mu-1)}\left[\mathrm{A}_{1}\left\|\mathrm{x}_{1}-\mathrm{x}_{2}\right\|_{0}+\mathrm{A}_{2}\left\|\mathrm{y}_{1}-\mathrm{y}_{2}\right\|_{0}\right]
\end{aligned}
$$




$$
\begin{aligned}
& +\frac{\mathcal{Z}_{\mu}}{\Gamma(\mu-1)}\left[\mathrm{A}_{1}\left\|\mathrm{x}_{1}-\mathrm{x}_{2}\right\|_{0}+\mathrm{A}_{2}\left\|\mathrm{y}_{1}-\mathrm{y}_{2}\right\|_{0}\right]+\frac{\mathcal{Z}_{\mu} \tau^{1-\omega}}{\Gamma(2-\omega)} \sum_{\mathrm{j}=1}^{\mathrm{m}} \check{\mathrm{L}}_{1 \mathrm{j}}\left\|\mathrm{x}_{1}-\mathrm{x}_{2}\right\|_{0} \\
& +\mathcal{Z}_{\mu} \sum_{\mathrm{j}=1}^{\mathrm{m}} \check{\mathrm{L}}_{1 \mathrm{j}}\left\|\mathrm{x}_{1}-\mathrm{x}_{2}\right\|_{0}+\frac{1}{2} \sum_{\mathrm{j}=1}^{\mathrm{m}} \check{\mathrm{L}}_{1 j}\left\|\mathrm{x}_{1}-\mathrm{x}_{2}\right\|_{0} \\
& \leq \frac{1}{\Gamma(\mu+1)}\left[\mathrm{A}_{1}+\mathrm{A}_{2}\right]\left\|\left(\mathrm{x}_{1}-\mathrm{x}_{2}, \mathrm{y}_{1}-\mathrm{y}_{2}\right)\right\|+\frac{1}{\Gamma(\mu+1)}\left[\mathrm{A}_{1}+\mathrm{A}_{2}\right]\left\|\left(\mathrm{x}_{1}-\mathrm{x}_{2}, \mathrm{y}_{1}-\mathrm{y}_{2}\right)\right\| \\
& +\frac{1}{\Gamma(\mu)}\left[\mathrm{A}_{1}+\mathrm{A}_{2}\right]\left\|\left(\mathrm{x}_{1}-\mathrm{x}_{2}, \mathrm{y}_{1}-\mathrm{y}_{2}\right)\right\|+\frac{1}{2 \Gamma(\mu-1)}\left[\mathrm{A}_{1}+\mathrm{A}_{2}\right]\left\|\left(\mathrm{x}_{1}-\mathrm{x}_{2}, \mathrm{y}_{1}-\mathrm{y}_{2}\right)\right\| \\
& +\sum_{j=1}^{m} L_{1 j}\left\|\left(x_{1}-x_{2}, y_{1}-y_{2}\right)\right\|_{0}+\sum_{j=1}^{m} \hat{L}_{1 j}\left\|\left(x_{1}-x_{2}, y_{1}-y_{2}\right)\right\| \\
& +\frac{\mathcal{Z}_{\mu}}{\Gamma(\mu-\omega+1)}\left[\mathrm{A}_{1}+\mathrm{A}_{2}\right]\left\|\left(\mathrm{x}_{1}-\mathrm{x}_{2}, \mathrm{y}_{1}-\mathrm{y}_{2}\right)\right\| \\
& +\frac{\mathcal{Z}_{\mu} \tau^{1-\omega}}{\Gamma(\mu) \Gamma(2-\omega)}\left[\mathrm{A}_{1}+\mathrm{A}_{2}\right]\left\|\left(\mathrm{x}_{1}-\mathrm{x}_{2}, \mathrm{y}_{1}-\mathrm{y}_{2}\right)\right\| \\
& +\frac{\mathcal{Z}_{\mu} \tau^{2-\omega}}{\Gamma(\mu-1) \Gamma(3-\omega)}\left[\mathrm{A}_{1}+\mathrm{A}_{2}\right]\left\|\left(\mathrm{x}_{1}-\mathrm{x}_{2}, \mathrm{y}_{1}-\mathrm{y}_{2}\right)\right\| \\
& +\frac{\mathcal{Z}_{\mu} \tau^{1-\omega}}{\Gamma(\mu-1) \Gamma(2-\omega)}\left[\mathrm{A}_{1}+\mathrm{A}_{2}\right]\left\|\left(\mathrm{x}_{1}-\mathrm{x}_{2}, \mathrm{y}_{1}-\mathrm{y}_{2}\right)\right\| \\
& +\frac{\mathcal{Z}_{\mu} \tau^{1-\omega}}{\Gamma(2-\omega)} \sum_{\mathrm{j}=1}^{\mathrm{m}} \hat{\mathrm{L}}_{1 \mathrm{j}}\left\|\left(\mathrm{x}_{1}-\mathrm{x}_{2}, \mathrm{y}_{1}-\mathrm{y}_{2}\right)\right\|+\frac{\mathcal{Z}_{\mu} \tau^{2-\omega}}{\Gamma(3-\omega)} \sum_{\mathrm{j}=1}^{\mathrm{m}} \check{\mathrm{L}}_{1 \mathrm{j}}\left\|\left(\mathrm{x}_{1}-\mathrm{x}_{2}, \mathrm{y}_{1}-\mathrm{y}_{2}\right)\right\| \\
& +\frac{\mathcal{Z}_{\mu}}{\Gamma(\mu-1)}\left[\mathrm{A}_{1}+\mathrm{A}_{2}\right]\left\|\left(\mathrm{x}_{1}-\mathrm{x}_{2}, \mathrm{y}_{1}-\mathrm{y}_{2}\right)\right\|+\frac{\mathcal{Z}_{\mu}}{\Gamma(\mu-1)}\left[\mathrm{A}_{1}+\mathrm{A}_{2}\right]\left\|\left(\mathrm{x}_{1}-\mathrm{x}_{2}, \mathrm{y}_{1}-\mathrm{y}_{2}\right)\right\| \\
& +\frac{\mathcal{Z}_{\mu} \tau^{1-\omega}}{\Gamma(2-\omega)} \sum_{\mathrm{j}=1}^{\mathrm{m}} \check{\mathrm{L}}_{1 j}\left\|\left(\mathrm{x}_{1}-\mathrm{x}_{2}, \mathrm{y}_{1}-\mathrm{y}_{2}\right)\right\|+\mathcal{Z}_{\mu} \sum_{\mathrm{j}=1}^{\mathrm{m}} \check{\mathrm{L}}_{1 \mathrm{j}}\left\|\left(\mathrm{x}_{1}-\mathrm{x}_{2}, \mathrm{y}_{1}-\mathrm{y}_{2}\right)\right\| \\
& +\frac{1}{2} \sum_{\mathrm{j}=1}^{\mathrm{m}} \check{\mathrm{L}}_{1 \mathrm{j}}\left\|\left(\mathrm{x}_{1}-\mathrm{x}_{2}, \mathrm{y}_{1}-\mathrm{y}_{2}\right)\right\| \\
& \leq\left[( \mathrm { A } _ { 1 } + \mathrm { A } _ { 2 } ) \left(\frac{2}{\Gamma(\mu+1)}+\frac{1}{\Gamma(\mu)}+\frac{1}{2 \Gamma(\mu-1)}+\frac{\mathcal{Z}_{\mu}}{\Gamma(\mu+\omega-1)}+\frac{\mathcal{Z}_{\mu} \tau^{1-\omega}}{\Gamma(\mu) \Gamma(2-\omega)}\right.\right. \\
& \left.+\frac{\mathcal{Z}_{\mu} \tau^{2-\omega}}{\Gamma(\mu-1) \Gamma(3-\omega)}+\frac{\mathcal{Z}^{1-\omega}}{\Gamma(\mu-1) \Gamma(2-\omega)}++\frac{2 \mathcal{Z}_{\mu}}{\Gamma(\mu-1)}\right) \\
& +\sum_{j=1}^{m} L_{1 j}+\sum_{j=1}^{m} \hat{\mathrm{L}}_{1 j}+\frac{\mathcal{Z}_{\mu} \tau^{1-\omega}}{\Gamma(2-\omega)} \sum_{j=1}^{m} \hat{\mathrm{L}}_{1 j}+\frac{\mathcal{Z}_{\mu} \tau^{2-\omega}}{\Gamma(3-\omega)} \sum_{j=1}^{m} \check{\mathrm{L}}_{1 j} \\
& \left.+\frac{\mathcal{Z}_{\mu} \tau^{1-\omega}}{\Gamma(2-\omega)} \sum_{\mathrm{j}=1}^{\mathrm{m}} \check{\mathrm{L}}_{1 \mathrm{j}}+\mathcal{Z}_{\mu} \sum_{\mathrm{j}=1}^{\mathrm{m}} \check{\mathrm{L}}_{1 \mathrm{j}}+\frac{1}{2} \sum_{\mathrm{j}=1}^{\mathrm{m}} \check{\mathrm{L}}_{1 \mathrm{j}}\right]\left\|\left(\mathrm{x}_{1}-\mathrm{x}_{2}, \mathrm{y}_{1}-\mathrm{y}_{2}\right)\right\| .
\end{aligned}
$$

Now

$$
\begin{aligned}
\nabla\left(\mathcal{T}_{1}\left(\mathrm{x}_{1}, \mathrm{y}_{1}\right), \mathcal{T}_{1}\left(\mathrm{x}_{2}, \mathrm{y}_{2}\right), \omega\right) & =\frac{\omega}{\omega+\left\|\mathcal{T}_{1}\left(\mathrm{x}_{1}, \mathrm{y}_{1}\right)-\mathcal{T}_{1}\left(\mathrm{x}_{2}, \mathrm{y}_{2}\right)\right\|} v \\
& \succeq \frac{\omega}{\omega+\left\|\left(\mathrm{x}_{1}-\mathrm{x}_{2}, \mathrm{y}_{1}-\mathrm{y}_{2}\right)\right\|} v
\end{aligned}
$$




$$
\begin{aligned}
& \geq \frac{\omega}{\omega+\left\|\left(\eta \mathrm{x}_{1}, \eta \mathrm{x}_{2}\right)\right\|} v \\
& =\min \left\{\frac{\omega}{\omega+\left\|\left(\eta \mathrm{x}_{1}, \eta \mathrm{x}_{2}\right)\right\|} v, \frac{\omega}{\omega+\left\|\left(\eta \mathrm{y}_{1}, \eta \mathrm{y}_{2}\right)\right\|} v\right\} \\
& \succeq \sqrt{\min \left\{\frac{\omega}{\omega+\left\|\left(\eta \mathrm{x}_{1}, \eta \mathrm{x}_{2}\right)\right\|} v, \frac{\omega}{\omega+\left\|\left(\eta \mathrm{y}_{1}, \eta \mathrm{y}_{2}\right)\right\|} v\right\}} \\
& =\sqrt{\min \left\{\nabla\left(\eta \mathrm{x}_{1}, \eta \mathrm{x}_{2}, \omega\right), \nabla\left(\eta \mathrm{y}_{1}, \eta \mathrm{y}_{2}, \omega\right)\right\}} .
\end{aligned}
$$

In the same fashion, we can obtain

$$
\begin{aligned}
& \left|\mathcal{T}_{2}\left(\mathrm{x}_{1}, \mathrm{y}_{1}\right)(\mathrm{t})-\mathcal{T}_{2}\left(\mathrm{x}_{2}, \mathrm{y}_{2}\right)(\mathrm{t})\right| \\
& \leq \mathcal{Z}_{\gamma}\left[( \mathrm { B } _ { 1 } + \mathrm { B } _ { 2 } ) \left(\frac{1}{\mathcal{Z}_{\gamma} \Gamma(\gamma+1)}+\frac{1}{\Gamma(\gamma-\xi+1)}+\frac{\epsilon^{1-\xi}}{\Gamma(\gamma) \Gamma(2-\xi)}\right.\right. \\
& \left.\quad+\frac{\epsilon^{2-\xi}}{\gamma(\gamma-1) \Gamma(3-\xi)}+\frac{\epsilon^{1-\xi}}{\Gamma(\gamma-1) \Gamma(2-\xi)}+\frac{2}{\Gamma(\gamma-1)}\right) \\
& \left.\quad+\frac{\epsilon^{1-\xi}}{\Gamma(2-\gamma)} \sum_{j=1}^{m} \hat{\mathrm{L}}_{2 j}+\frac{\epsilon^{1-\xi}}{\Gamma(3-\gamma)} \sum_{\mathrm{j}=1}^{\mathrm{m}} \check{\mathrm{L}}_{2 \mathrm{j}}+\frac{\epsilon^{1-\xi}}{\Gamma(2-\gamma)} \sum_{\mathrm{j}=1}^{\mathrm{m}} \check{\mathrm{L}}_{2 \mathrm{j}}+\sum_{\mathrm{j}=1}^{\mathrm{m}} \check{\mathrm{L}}_{2 \mathrm{j}}\right] \\
& \quad \times\left\|\left(\mathrm{x}_{1}-\mathrm{x}_{2}, \mathrm{y}_{1}-\mathrm{y}_{2}\right)\right\|, \quad \mathrm{t} \in\left[0, \mathrm{t}_{1}\right]
\end{aligned}
$$

and

$$
\begin{aligned}
& \left|\mathcal{T}_{2}\left(\mathrm{x}_{1}, \mathrm{y}_{1}\right)(\mathrm{t})-\mathcal{T}_{2}\left(\mathrm{x}_{2}, \mathrm{y}_{2}\right)(\mathrm{t})\right| \\
& \leq\left[( \mathrm { B } _ { 1 } + \mathrm { B } _ { 2 } ) \left(\frac{2}{\Gamma(\gamma+1)}+\frac{1}{\Gamma(\gamma)}+\frac{1}{2 \Gamma(\gamma-1)}+\frac{\mathcal{Z}_{\gamma}}{\Gamma(\gamma-\xi+1)}+\frac{\mathcal{Z}_{\gamma} \epsilon^{1-\xi}}{\Gamma(\gamma) \Gamma(2-\xi)}\right.\right. \\
& \left.\quad+\frac{\mathcal{Z}_{\gamma} \epsilon^{2-\xi}}{\gamma(\gamma-1) \Gamma(3-\xi)}+\frac{\mathcal{Z}_{\gamma} \epsilon^{1-\xi}}{\Gamma(\gamma-1) \Gamma(2-\xi)}+\frac{2 \mathcal{Z}_{\gamma}}{\Gamma(\gamma-1)}\right)+\sum_{\mathrm{j}=1}^{\mathrm{m}} \mathrm{L}_{2 j}+\sum_{\mathrm{j}=1}^{\mathrm{m}} \hat{\mathrm{L}}_{2 \mathrm{j}} \\
& \quad+\frac{\mathcal{Z}_{\gamma} \epsilon^{1-\xi}}{\Gamma(2-\gamma)} \sum_{\mathrm{j}=1}^{\mathrm{m}} \hat{\mathrm{L}}_{2 \mathrm{j}}+\frac{\mathcal{Z}_{\gamma} \epsilon^{2-\xi}}{\Gamma(3-\gamma)} \sum_{\mathrm{j}=1}^{\mathrm{m}} \check{\mathrm{L}}_{2 \mathrm{j}}+\frac{\mathcal{Z}_{\gamma} \epsilon^{1-\xi}}{\Gamma(2-\gamma)} \sum_{\mathrm{j}=1}^{\mathrm{m}} \check{\mathrm{L}}_{2 \mathrm{j}}+\mathcal{Z}_{\gamma} \sum_{\mathrm{j}=1}^{\mathrm{m}} \check{\mathrm{L}}_{2 \mathrm{j}} \\
& \left.\quad+\frac{1}{2} \mathcal{Z}_{\gamma} \sum_{\mathrm{j}=1}^{\mathrm{m}} \check{\mathrm{L}}_{2 \mathrm{j}}\right] \times\left\|\left(\mathrm{x}_{1}-\mathrm{x}_{2}, \mathrm{y}_{1}-\mathrm{y}_{2}\right)\right\|, \quad \mathrm{t} \in\left[\mathrm{t}_{\mathrm{m}}, \mathrm{t}_{\mathrm{t}+1}\right] .
\end{aligned}
$$

Similarly, one can show that

$$
\nabla\left(\mathcal{T}_{2}\left(\mathrm{x}_{1}, \mathrm{y}_{1}\right), \mathcal{T}_{2}\left(\mathrm{x}_{2}, \mathrm{y}_{2}\right), \omega\right) \succeq \sqrt{\min \left\{\nabla\left(\eta \mathrm{x}_{1}, \eta \mathrm{x}_{2}, \omega\right), \nabla\left(\eta \mathrm{y}_{1}, \eta \mathrm{y}_{2}, \omega\right)\right\}}
$$

Thus, from (4.2) -(4.9), we infer that all conditions of Corollary 3.3 are satisfied. Hence $\mathcal{T}$ has a fixed point $\left(\mathrm{x}^{*}(\mathrm{t}), \mathrm{y}^{*}(\mathrm{t})\right) \in \mathcal{X}^{\prime}$, which is unique. Therefore, problem (1.1) has a unique solution $\left(\mathrm{x}^{*}(\mathrm{t}), \mathrm{y}^{*}(\mathrm{t})\right)$.

\section{Conclusion}

Due to the many applications in which the fixed point method is involved, this is an important pillar and a good tool in nonlinear analysis. This technique mainly participates in 
the complex analysis in terms of studying the existence and uniqueness of the FP within the various spaces that include complex numbers such as complex-valued metric space, complex-valued $b$-metric space, etc. In the setting of the fuzzy set framework, this formalism is devoted to obtaining the FP for single and multivalued mappings via suitable conditions. Nonlinear analysis and fixed point theory play a prominent role in many fields of mathematics, and by the technique of FP, we can solve several existing problems in mathematics. Blood flow systems, aerodynamics, the nonlinear oscillation of earthquake, the fluid-dynamic traffic model, and control theory both can be studied mathematically by fractional calculus. Because of that, this discipline has turned around many researchers and readers especially when we deal with these problems in a fixed point fashion. One of the important branches of fractional calculus is impulsive fractional differential equations since the pulse effect is significant in many processes and phenomena. For example, in biological systems such as heartbeats, blood flows, mechanical systems with impact, population dynamical systems, and so on. By successful applications of our derived results, we have studied the existence and uniqueness solutions for a coupled system of impulsive fractional differential equations via coupled FP techniques in the setting of CVFMSs. In addition, some theoretical results and nontrivial examples have been also presented.

Acknowledgements

The authors are grateful to the Spanish Government and the European Commission for Grant IT1207-19.

Funding

This work was supported in part by the Basque Government under Grant IT1207-19.

Availability of data and materials

Not applicable.

Competing interests

The authors declare that they have no competing interests.

Authors' contributions

All authors contributed equally and significantly in writing this article. All authors read and approved the final manuscript.

Author details

'Department of Mathematics, University of Malakand, Chakdara Dir(L), Pakistan. ${ }^{2}$ Department of Mathematics, Faculty of Science, Sohag University, Sohag 82524, Egypt. ${ }^{3}$ Institute of Research and Development of Processes, University of the Basque Country, 48940 Leioa (Bizkaia), Spain.

\section{Publisher's Note}

Springer Nature remains neutral with regard to jurisdictional claims in published maps and institutional affiliations.

Received: 2 March 2021 Accepted: 27 April 2021 Published online: 07 May 2021

References

1. Zadeh, L.A.: Fuzzy sets. Inf. Control 8, 338-353 (1965)

2. Heilpern, S.: Fuzzy mappings and fixed point theorem. J. Math. Anal. Appl. 83(2), 566-569 (1981)

3. Choudhury, B.S., Das, K., Das, P.: Coupled coincidence point results for compatible mappings in partially ordered fuzzy metric spaces. Fuzzy Sets Syst. 222(1), 84-97 (2013)

4. Ćirić, L.: Some new results for Banach contractions and Edelstein contractive mappings on fuzzy metric spaces. Chaos Solitons Fractals 42(1), 146-154 (2009)

5. Mihet, D.: On fuzzy contractive mappings in fuzzy metric spaces. Fuzzy Sets Syst. 158, 915-921 (2007)

6. Saha, P., Choudhury, B.S., Das, P.: A new contractive mapping principle in fuzzy metric spaces. Bull dell'Uni Math Ital. 8(4), 287-296 (2016)

7. Kaleva, O., Seikkala, S.: On fuzzy metric spaces. Fuzzy Sets Syst. 12, 215-229 (1984)

8. Kramosil, I., Michalek, J.: Fuzzy metric and statistical metric spaces. Kybernetica 11, 336-344 (1975)

9. George, A., Veeremani, P.: On some results of analysis for fuzzy metric spaces. Fuzzy Sets Syst. 90, 365-368 (1994)

10. Došenović, T., Rakić, D., Brdar, M.: Fixed point theorem in fuzzy metric spaces using altering distance. Filomat 28(7), 1517-1524 (2014)

11. Gregori, V., Sapena, A.: On fixed-point theorem in fuzzy metric spaces. Fuzzy Sets Syst. 125, 245-252 (2002)

12. Mihet, D.: A Banach contraction theorem in fuzzy metric spaces. Fuzzy Sets Syst. 144, 431-439 (2004) 
13. Sedghi, S., Shobe, N., Selahshoor, M.A.: A common fixed point theorem for four mappings in two complete fuzzy metric spaces. Adv. Fuzzy Math. 1(1) (2006)

14. Sedghi, S., Shobkolaei, N., Došenović, T., Radenović, S.: Suzuki-type of common fixed point theorems in fuzzy metric spaces. Math. Slovaca 68(2), 451-462 (2018)

15. Wairojjana, N., Došenović, T., Rakić, D., Gopal, D., Kumam, P.: An altering distance function in fuzzy metric fixed point theorems. Fixed Point Theory Appl. 2015, 69 (2015)

16. Azam, A., Fisher, B., Khan, M.: Common fixed point theorems in complex-valued metric spaces. Numer. Funct. Anal. Optim. 32, 243-253 (2011)

17. Shukla, S., Rodríguez-Lopez, R., Abbas, M.: Fixed point results for contractive mappings in complex-valued fuzzy metric spaces. Fixed Point Theory 19(2), 1-22 (2018)

18. Chandok, S., Mukheimer, A., Hussain, A., Paunovic, L.: Picard-Jungck operator for a pair of mappings and simulation type functions. Mathematics 7, 5 (2019)

19. Hussain, A., Adeel, M.: Remarks on new fixed point theorems for contractions in ordered metric spaces. Fixed Point Theory Appl. 21(2) (2019)

20. Hassan, S., De la Sen, M., Agarwal, P., Ali, Q., Hussain, A.: A new faster iterative scheme for numerical fixed points estimation of Suzuki's generalized nonexpansive mappings. Math. Probl. Eng. 2020, Article ID 3863819 (2020). https://doi.org/10.1155/2020/3863819

21. Agarwal, P., Jleli, M., Samet, B.: Fixed Point Theory in Metric Spaces, Recent Advances and Applications. Springer, Berlin (2018)

22. Joshi, V., Jain, S.: G-Metric Spaces: From the Perspective of F-Contractions and Best Proximity Points, Page 103, Metric Structures and Fixed Point Theory. CRC Press, Boca Raton (2022)

23. Gopal, D., Jain, S.: Fixed Point Theory in Partial Metric Spaces, Pages 282, Metric Structures and Fixed Point Theory. CRC Press, Boca Raton (2022)

24. Gopal, D., Budhia, L.M., Jain, S.: A relation theoretic approach for $\phi$-fixed point result in metric space with an application to an integral equation. Commun. Appl. Nonlinear Anal. 6(1), 89-95 (2019)

25. Hammad, H.A., De la Sen, M.: A coupled fixed point technique for solving coupled systems of functional and nonlinear integral equations. Mathematics 7, 634 (2019)

26. Brikaa, M.: Existence results for a couple system of nonlinear fractional differential equation with three point boundary conditions. J. Fract. Calc. Appl. 3, 1-10 (2015)

27. Henderson, J., Luca, R.: Positive solutions for a system of fractional differential equations with coupled integral boundary conditions. Appl. Math. Comput. 249, 182-197 (2014)

28. Podlubny, l.: Fractional Differential Equations. Academic Press, San Diego (1999)

29. Baleanu, D., Etemad, S., Rezapour, S.: A hybrid Caputo fractional modeling for thermostat with hybrid boundary value conditions. Bound. Value Probl. 2020, 64 (2020)

30. Thabet, S.T.M., Etemad, S., Rezapour, S.: On a coupled Caputo conformable system of pantograph problems. Turk. J. Math. 45(1), 496-519 (2021)

31. Mohammadi, H., Kumar, S., Rezapour, S., Etemad, S.: A theoretical study of the Caputo-Fabrizio fractional modeling for hearing loss due to Mumps virus with optimal control. Chaos Solitons Fractals 144, 110668 (2021)

32. Thabet, S.T.M., Etemad, S., Rezapour, S.: On a new structure of the pantograph inclusion problem in the Caputo conformable setting. Bound. Value Probl. 2020, 171 (2020)

33. Qi, T., Liu, Y., Zou, Y.: Existence result for a class of coupled fractional differential systems with integral boundary value conditions. J. Nonlinear Sci. Appl. 10, 4034-4045 (2017)

34. Zhang, Y.: Existence results for a coupled system of nonlinear fractional multi-point boundary value problems at resonance. J. Inequal. Appl. 2018, 198 (2018)

35. Zhang, Y., Bai, Z., Feng, T.: Existence results for a coupled system of nonlinear fractional three-point boundary value problems at resonance. Comput. Math. Appl. 61, 1032-1047 (2011)

36. Zada, A., Ali, Z., Xu, J., Cul, Y.: Stability results for a coupled system of impulsive fractional differential equations. Mathematics 7, 927 (2019)

37. Hammad, H.A., Aydi, H., De la Sen, M.: Solutions of fractional differential type equations by fixed point techniques for multi-valued contractions. Complixty 2021, Article ID 5730853 (2021). https://doi.org/10.1155/2021/5730853

38. Hammad, H.A., De la Sen, M.: Tripled fixed point techniques for solving system of tripled-fractional differential equations. AlMS Math. 6(3), 2330-2343 (2020)

39. Hammad, H.A., Aydi, H., Mlaiki, N.: Contributions of the fixed point technique to solve the 2D Volterra integral equations, Riemann-Liouville fractional integrals, and Atangana-Baleanu integral operators. Adv. Differ. Equ. 2021, 97 (2021)

40. Fu, X., Bao, X.: Some existence results for nonlinear fractional differential equations with impulsive and fractional integral boundary conditions. Adv. Differ. Equ. 2014, 129 (2014)

41. Yukunthorn, W., Ahmad, B., Ntouyas, S., Tariboon, J: On Caputo-Hadamard type fractional impulsive hybrid systems with nonlinear fractional integral conditions. Nonlinear Anal. Hybrid Syst. 19, 77-92 (2016)

42. Zhao, K., Liang, J.: Solvability of triple-point integral boundary value problems for a class of impulsive fractional differential equations. Adv. Differ. Equ. 2017, 50 (2017)

43. Lakshmikantham, V., Cirić, Lj.B.: Coupled fixed point theorems for nonlinear contractions in partially ordered metric spaces. Nonlinear Anal. 70, 4341-4349 (2009) 